\title{
Hippo Signaling Pathway Has a Critical Role in Zika Virus Replication and in the Pathogenesis of Neuroinflammation
}

Gustavo Garcia, Jr, * Sayan Paul, ${ }^{\dagger}$ Sara Beshara, * V. Krishnan Ramanujan, ${ }^{\ddagger}$ Arunachalam Ramaiah, ${ }^{\S}$ Karin Nielsen-Saines, Melody M.H. Li, Samuel W. French, ${ }^{* *}$ Kouki Morizono, ${ }^{\dagger \dagger \ddagger}$ Ashok Kumar, ${ }^{\S \S}$ and Vaithilingaraja Arumugaswami ${ }^{* \pm \uparrow \Phi}$

From the Departments of Molecular and Medical Pharmacology,* Microbiology, Immunology, and Molecular Genetics, ${ }^{\|}$Pathology and Laboratory

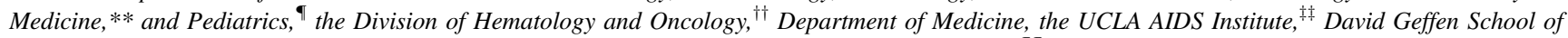
Medicine, and the Eli and Edythe Broad Center of Regenerative Medicine and Stem Cell Research, ${ }^{\text {甲 }}$ University of California, Los Angeles, California; the Department of Biotechnology, ${ }^{\dagger}$ Manonmaniam Sundaranar University, Tirunelveli, India; the Department of Surgery, ${ }^{\ddagger}$ Cedars-Sinai Medical Center, Los Angeles, California; the Department of Ecology and Evolutionary Biology, ${ }^{\S}$ University of California, Irvine, California; and the Department of Ophthalmology, Visual and Anatomical Sciences, ${ }^{\S \S}$ Wayne State University, Detroit, Michigan

\author{
Accepted for publication \\ December 17, 2019. \\ Address correspondence to \\ Vaithilingaraja Arumu- \\ gaswami, D.V.M., Ph.D., \\ 10833 Le Conte Ave., CHS \\ B2-049A, Los Angeles, CA \\ 90095. E-mail: \\ varumugaswami@mednet. \\ ucla.edu.
}

\begin{abstract}
Zika virus (ZIKV) is a reemerging human pathogen that causes congenital abnormalities, including microcephaly and eye disease. The cellular/molecular basis of ZIKV and host interactions inducing ocular and neuronal pathogenesis are unclear. Herein, we noted that the Hippo/Salvador-Warts-Hippo signaling pathway, which controls organ size through progenitor cell proliferation and differentiation, is dysregulated after ZIKV infection. In human fetal retinal pigment epithelial cells, there is an early induction of transcriptional coactivator, Yes-associated protein (YAP), which is later degraded with a corresponding activation of the TANK binding kinase 1/interferon regulatory factor 3 type I interferon pathway. YAP/transcriptional co-activator with a PDZ-binding domain (TAZ) silencing results in reduced ZIKV replication, indicating a direct role of Hippo pathway in regulating ZIKV infection. Using an in vivo Ifnar1 $^{-/-}$knockout mouse model, ZIKV infection was found to reduce YAP/TAZ protein levels while increasing phosphorylated YAP Ser127 in the retina and brain. Hippo pathway is activated in major cellular components of the blood-brain barrier, including endothelial cells and astrocytes. In addition, this result suggests AMP-activated protein kinase signaling pathway's role in regulating YAP/TAZ in ZIKV-infected cells. These data demonstrate that ZIKV infection might initiate a cross talk among AMP-activated protein kinase-Hippo-TBK1 pathways, which could regulate antiviral and energy stress responses during oculoneuronal inflammation. (Am J Pathol 2020, 190: 844-861; https://doi.org/ 10.1016/j.ajpath.2019.12.005)
\end{abstract}

Zika virus (ZIKV) is a mosquito-borne positive-sense RNA virus that was first isolated in 1947 in Uganda, Africa. Since then, there have been numerous outbreaks throughout Africa and Asia, and, most recently, the pandemic in the Americas and Caribbean islands. ${ }^{1}$ In 2016, the World Health Organization declared a global health emergency because of an increasing number of microcephaly cases and other neurologic-ocular disorders ${ }^{2,3}$ reported in South America. Studies demonstrated that vertical transmission of ZIKV in infected mothers causes microcephaly, fetal growth restriction, and ocular abnormalities in developing fetuses. ${ }^{4}$
Involvement of ZIKV in the eye was reported in several cases from Brazil and other affected countries, where infants born with microcephaly showed evidence of retinal lesions, focal pigmented mottling, optic nerve abnormalities,

\footnotetext{
Supported by the California Institute for Regenerative Medicine Quest-Discovery Stage Research Projects grant DISC2-10188 (V.A.), NIH award R21AI135583 (A.K.), and NIH grants R21AI095004 (K.M.) and R01AI108400 (K.M.).

G.G. and S.P. contributed equally to this work.

Disclosures: None declared.
} 
chorioretinal atrophy, and a withering of the retina and choroid. ${ }^{2,5-7}$ There might be more cases of ZIKV-mediated neural and ocular complications because many are underreported. ${ }^{8}$ ZIKV infection in adults is linked to GuillainBarre syndrome and neurologic complications, including encephalitis/meningoencephalitis, myelitis, and acute disseminated encephalomyelitis. ${ }^{9,10}$ These inflammatory changes and cerebrovascular complications can lead to seizures and encephalopathy, sensory polyneuropathy, and sensory neuropathy. The recent ZIKV outbreaks and associated neurologic disorders generated a need to understand the disease pathology and molecular mechanisms for development of effective ZIKV vaccine and therapeutic agents. ${ }^{3,9,11-14}$ However, the molecular mechanism underlying ZIKV infection is not well understood.

Recently, in vitro models of ZIKV infection ${ }^{12,15,16}$ have demonstrated that ZIKV infects retinal cells and cells lining the blood-retinal barrier, including retinal pigment epithelial (RPE) cells and retinal vascular endothelium. The RPE constitutes the outer blood-retinal barrier and shields the neuroretina from hematogenous pathogens, including ZIKV. Therefore, disruption of RPE could generate a route of entry for ZIKV through the fenestrated choroidal capillaries. Similarly, the remarkable neurotropism of ZIKV indicates the virus has tremendous ability to infiltrate the blood-brain barrier (BBB). Different cell types, such as endothelial cells, astrocytes, pericytes, and microglia, compose the BBB. ${ }^{17,18}$ Studies have shown that the AXL receptor tyrosine kinase (AXL) protein is enriched in endothelial cells, radial glial cells, astrocytes, and neural stem cells, which mediate ZIKV entry. ${ }^{19}$ ZIKV can infect human brain microvascular endothelial cells and pericytes. ${ }^{16,20}$ Moreover, astrocytes are critical in preserving the BBB in addition to maintaining homeostasis and providing energy and metabolic support to neurons. ${ }^{21,22}$ Therefore, on ZIKV infection, the release of inflammatory mediators and influx of immune and inflammatory cells can disrupt the endothelial integrity and affect the BBB.

Mice models have been useful in investigating the ZIKV disease pathogenesis mechanism. ${ }^{23}$ Several studies have used interferon (IFN) receptor knockout $\left(\right.$ Ifnarl $^{-1-}$ ) mice for systemic or direct inoculation of ZIKV into the desired tissue/organ. ${ }^{11,17,23-26}$ The innate and specific immune responses critical for controlling ZIKV infection have been extensively investigated. ${ }^{27-30}$ Ifnarl $^{-1-}$ infected mice showed neutrophil and macrophage infiltrates in the central nervous system with high expression levels of IL-1, IL-6, and cyclooxygenase 2 (COX2). ZIKV infection has been shown to elicit epitope-specific polyfunctional cytotoxic $\mathrm{CD}^{+}{ }^{\mathrm{T}}$ cells. ${ }^{11,12,31-34}$ Thus, the mouse model is relevant for understanding the pathogenesis of ZIKV disease.

Hippo signaling pathway is shown to control neural progenitor cell proliferation and differentiation and organ size. ${ }^{35,36}$ Because ZIKV affects the developing eye and brain, we hypothesized that the developmentally conserved Hippo pathway could be dysregulated during ZIKV infection. The key components of the Hippo pathway in mammals are STE20-like (serine/threonine) kinases (MST1/2; Hpo in Drosophila) and large tumor suppressor (LATS1/2; Wts in Drosophila). On activation by stimuli, MST1/2 phosphorylate and activate the LATS1/2. The activated phosphorylated LATS1/2, along with Salvador 1, then phosphorylates YY1 associated protein 1 (YY1AP1), also known as Yesassociated protein (YAP; oncogene and a transcription coactivator) at serine 127 residue ${ }^{37}$ The phosphorylated YAP S127, then bound to a cytoplasmic 14-3-3 protein, results in nuclear exclusion. Subsequent YAP phosphorylation at serine 397 by casein kinase $1 \delta$ primes it for ubiquitination and degradation by proteasomes. ${ }^{36}$ While Hippo pathway is inactive, the unphosphorylated YAP and its homologous partner, tafazzin, also known as transcriptional coactivator with PDZ-binding motif [transcriptional co-activator with a PDZ-binding domain (TAZ) or WW domain containing transcription regulator 1 (WWTR1)], translocate to the nucleus, where they bind to the TEA domain transcription factor (TEAD) family of transcription factors and mediate expression of target genes (BIRC5, CTCF, AXIN2, MYC, and $C C N D 1)$ that are involved in cell survival and proliferation. Recent evidence suggests that the Hippo pathway modulates host antiviral immune responses, ${ }^{38-41}$ where YAP inhibits antiviral defense mechanisms by antagonizing the function of proinnate immune factors TBK1 and IRF3. ${ }^{42}$ YAP depletion can relieve TBK1 and IRF3 suppression by YAP, ${ }^{42}$ resulting in antiviral response by stimulating the production of type I and III interferons.

In this study, in vitro and in vivo models were used to characterize how ZIKV modulates the Hippo signaling pathway. We found that the level of YAP/TAZ changes during ZIKV infection because of Hippo pathway activation. shRNA knockdown showed that YAP/TAZ plays a direct role in ZIKV replication. Lastly, investigating the interplay between the host cell and ZIKV in mouse model revealed that the activation of Hippo pathway targets BBB and immune and inflammatory cells, contributing to energy stress, antiviral response, and neuroinflammation.

\section{Materials and Methods}

\section{Ethics Statement}

The Institutional Animal Care Use Committee of the University of California, Los Angeles (UCLA) and CedarsSinai Medical Center have provided approval of the study. This work was performed in strict compliance with the recommendations of NIH's Guide for the Care and Use of Laboratory Animals. ${ }^{43}$

\section{Cell Lines}

Human fetal RPE cells were obtained from Dr. Guoping Fan (UCLA). The cells were collected with required informed consent described in earlier work. ${ }^{44,45}$ Fetal retinas 
Table 1 List of Antibodies Used in This Study

\begin{tabular}{|c|c|c|}
\hline Antibodies & Source & Catalog no. \\
\hline Acetyl-CoA carboxylase (C83B10) rabbit mAb & Cell Signaling (Danvers, MA) & 3676 \\
\hline AMPK $\alpha$ (D5A2) rabbit mAb & Cell Signaling & 5831 \\
\hline Anti-IRF3 (phospho S386) antibody (EPR2346) & Abcam & ab76493 \\
\hline CD45 antibody (YW62.3) & Bio-Rad (Hercules, CA) & MCA1031GA \\
\hline Cleaved caspase- 3 rabbit monoclonal antibody, clone D175 & Cell Signaling & $9661 S$ \\
\hline Donkey anti-rat Cy3 & Jackson ImmunoResearch & $712-165-153$ \\
\hline Goat anti-Armenian hamster alexa fluor-488 & Abcam & ab173003 \\
\hline Goat anti-mouse alexa flour-555 & Thermo Fisher Scientific & A32727 \\
\hline Goat anti-rabbit alexa flour- 488 & Thermo Fisher Scientific & A-11008 \\
\hline Goat anti-rabbit alexa fluor- 647 & Thermo Fisher Scientific & A32733 \\
\hline Monoclonal anti- $\beta$-actin antibody produced in mouse & Sigma-Aldrich & A5316 \\
\hline Mouse anti-occludin (OC-3F10), monoclonal & Thermo Fisher Scientific & $33-1500$ \\
\hline MST1 antibody & Cell Signaling & 3682 \\
\hline NeuN rabbit monoclonal antibody, clone D4G40 & Cell Signaling & $24307 S$ \\
\hline NK1.1 monoclonal antibody (PK136), eBioscience & Thermo Fisher Scientific & $14-5941-82$ \\
\hline Phospho-acetyl-CoA carboxylase (Ser79) antibody number 3661 & Cell Signaling & $3661 \mathrm{~s}$ \\
\hline Phospho-AMPK $\alpha$ (Thr172) (40H9) rabbit mAb number 2535 & Cell Signaling & $2535 T$ \\
\hline Phospho-AMPK $\alpha$ (Thr172) (D4D6D)rabbit mAb & Cell Signaling & 50081 \\
\hline Phospho-IRF-3 (Ser386) (E7J8G) XP rabbit mAb & Cell Signaling & 37829 \\
\hline Phospho-stat1 (Tyr701) (58D6) rabbit mAb number 9167 & Cell Signaling & $9167 \mathrm{~S}$ \\
\hline Phospho-TBK1/NAK (Ser172) (D52C2) XP rabbit mAb & Cell Signaling & $5483 S$ \\
\hline Stat1 (D1K9Y) Rabbit mAb & New England Biolabs (Ipswich, MA) & 14994 \\
\hline TBK1/NAK (E9H5S) mouse mAb & Cell Signaling & $51872 S$ \\
\hline Polyclonal rabbit anti-GFAP & Agilent Dako (Santa Clara, CA) & Z033429-2 \\
\hline Purified Armenian hamster anti-CD11C, clone N418 & BioLegend (San Diego, CA) & 117302 \\
\hline Zika virus NS1 protein antibody & Genetex (Irvine, CA) & GTX133307 \\
\hline Zika virus NS4B protein antibody, rabbit polyclonal & GeneTex & GTX133311 \\
\hline
\end{tabular}

AMPK $\alpha$, AMP-activated protein kinase $\alpha$; GFAP, glial fibrillary acidic protein; HRP, horseradish peroxidase; IRF3, interferon regulatory factor 3; LATS, large tumor suppressor; mAb, monoclonal antibody; MST, STE20-like (serine/threonine) kinase; NeuN, neuronal nuclei; NS, non-structural; TBK1, TANK binding kinase 1; TAZ, ranscriptional o-activator with PDZ-binding motif; YAP, Yes-associated protein.

(approximately 20 weeks old) were used for isolating RPEs at the laboratory of Dr. Fan using a previously reported protocol. $^{44,45}$ The cells were cultured in minimal essential medium- $\alpha$ modification medium containing $10 \%$ fetal bovine serum, $1 \%$ penicillin/streptomycin, $1 \%$ N2 supplement, $1 \%$ glutamine, $125 \mathrm{mg}$ taurine, $1 \%$ nonessential amino acids, $10 \mu \mathrm{g}$ hydrocortisone, and $6.5 \mathrm{ng}$ triiodothyronine. Cells of early passage (4 to 8 ) were used.

The human TIM-1 gene was cloned into the lentiviral vector plasmid, pLenti CMV Puro DEST (Addgene,
Cambridge, MA). ${ }^{46}$ The TIM-1-expressing lentiviral vector was produced by calcium phosphate transfection method. ${ }^{47}$ TIM-HEK-1 cells were generated by transducing HEK293 cells (ATCC, Manassas, VA) with lentiviral vectors expressing TIM-1, followed by culture in Iscove's modified Dulbecco's medium (Sigma-Aldrich, St. Louis, MO) supplemented with $10 \%$ fetal calf serum (Sigma-Aldrich) and $1 \mu \mathrm{g} / \mathrm{mL}$ puromycin (Life Technologies, Camarillo, CA). Human glioblastoma cell line U-87 MG and Vero cell line were purchased from ATCC. The cells were incubated at 
A

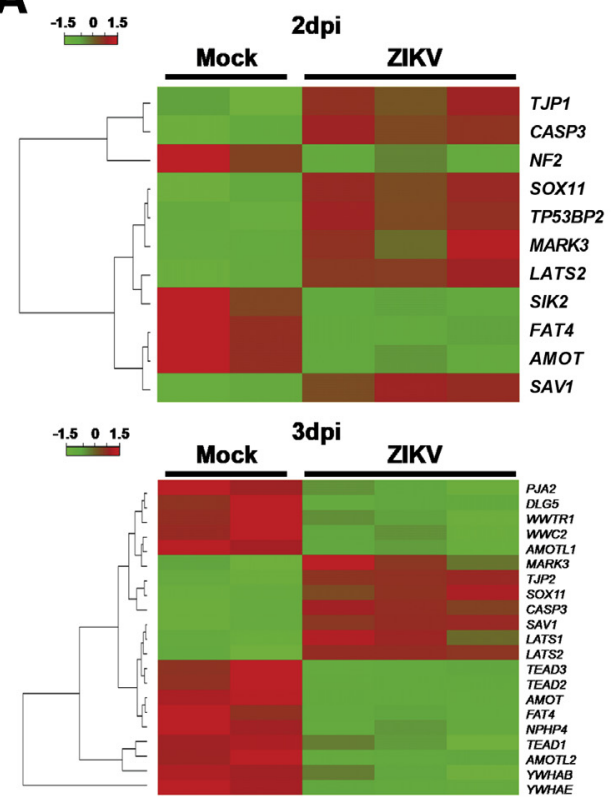

B
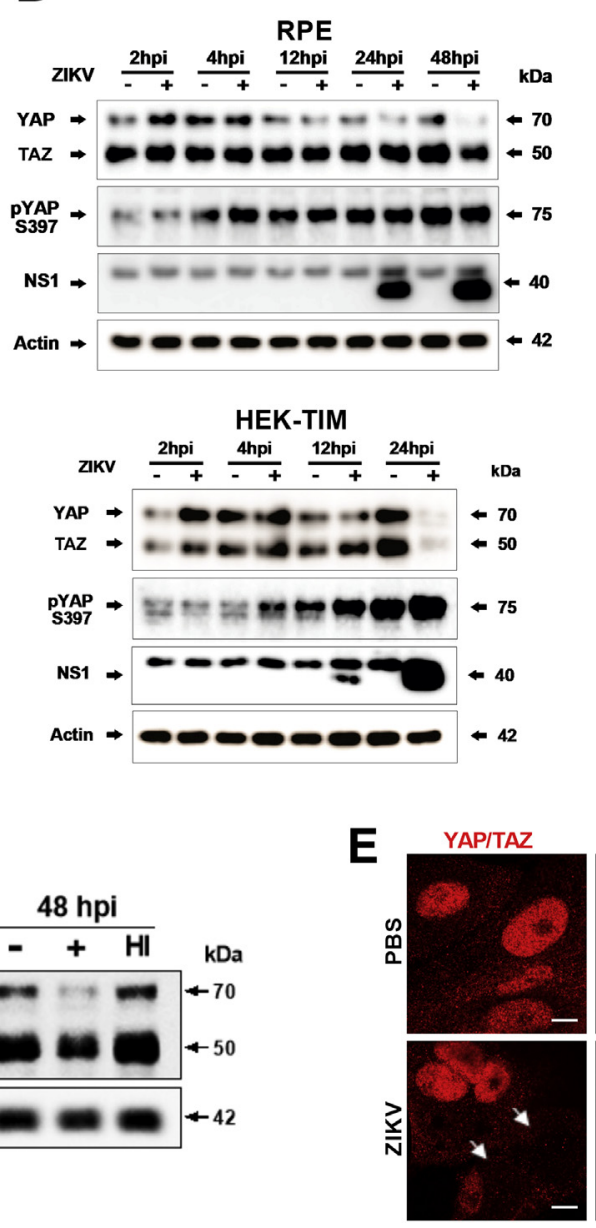

C
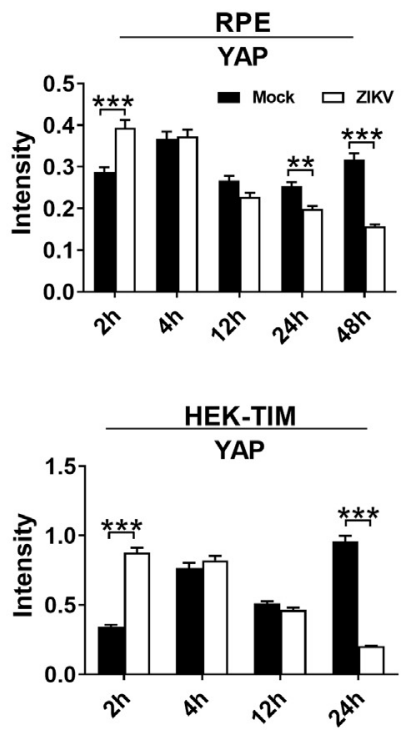

RPE

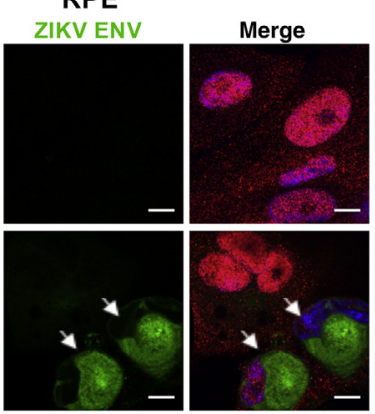

Figure 1 Zika virus (ZIKV) infection dysregulates Hippo signaling pathway. A: Heat maps show differentially expressed Hippo pathway genes at 2 and 3 days post infection (dpi) in ZIKV-infected retinal pigment epithelial (RPE) cells. ZIKV infections were done in biological triplicates for each time point. The uninfected controls were done in biological quadruplicates and pooled in duplicates for downstream analyses. B: Western blot analysis of Hippo pathway. C: Graphs showing $\beta$-actin-normalized Western blot signal intensity for YAP protein are presented. Representative data from three independent experiments are presented. D: Kinetics of viral production by infected RPE cells at indicated time points after infection. Viral titer was measured by plaque assay using Vero cells. Heat-inactivated (HI) ZIKV has not yielded infectious virus, and not degraded YAP/TAZ, as determined by Western blot analysis. Dashed line represents the assay detection limit. E: Immunohistochemistry images reveal a reduction in YAP/TAZ protein level in infected cells (arrowheads) at 24 hours postinfection (hpi). ${ }^{* * P}<0.01,{ }^{* *} P<0.001$ (t-test). Scale bars $=10 \mu \mathrm{m}(\mathrm{E}) . \mathrm{M}$, mock; PBS, phosphate-buffered saline; PFU, plaque-forming unit; TAZ, transcriptional co-activator with a PDZ-binding domain.

$37^{\circ} \mathrm{C}$, supplemented with $5 \% \mathrm{CO}_{2}$, and subcultured when $90 \%$ confluence was reached at approximately every second to third day using $0.05 \%$ trypsin plus $0.53 \mathrm{mmol} / \mathrm{L}$ EDTA (Corning, Corning, NY). Use of these established cell lines for the study was approved by the Institutional Biosafety Committee of Cedars-Sinai Medical Center and UCLA.

\section{shRNA-Mediated Gene Silencing}

HEK-TIM-1 cells $\left(2.5 \times 10^{4}\right.$ cells/well $)$ were added in a 48-well plate. After 24 hours, the pLKO.1-puro shRNA targeting YAP/TAZ (5'-CCGGCCCAGTTAAATGTTCACCAATCTCGAGATTGGTGAACATTTAACTGGGT TTTTG-3') or pLKO.1-puro Non-Targeting shRNA Control plasmid (Sigma-Aldrich) was transfected using Lipofectamine 2000 (Invitrogen, Waltham, MA). At 48 hours after transfection, ZIKV with a multiplicity of infection of 1.0 was added. At 24 hours after infection, cell culture supernatant and cell protein lysates were collected for virus titer and YAP/TAZ protein knockdown efficiency assessments.

\section{ZIKV}

Asian genotype Zika virus strain, PRVABC59 (https://www. ncbi.nlm.nih.gov; GenBank accession number KU501215), was used in this study. PRVABC59 was acquired from the CDC (Atlanta, GA). Viral stocks were generated in Vero cells. Viral titer was measured by plaque assay, as previously described. ${ }^{48}$ For heat inactivation, ZIKV was subjected to heat treatment for 1 hour at $70^{\circ} \mathrm{C}$. 
A

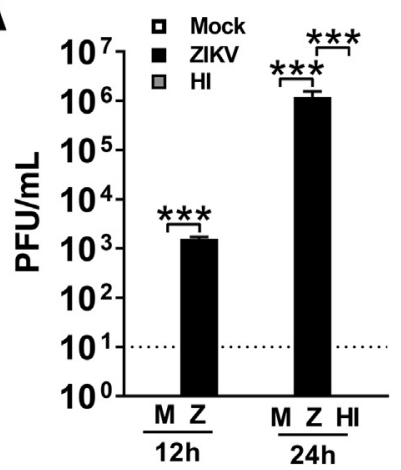

B
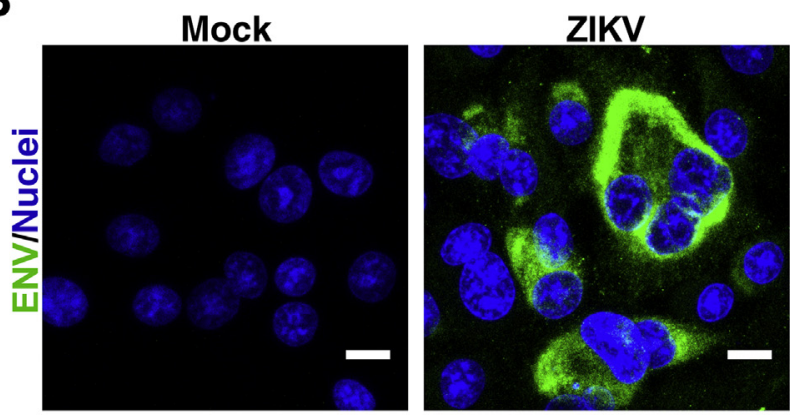

D

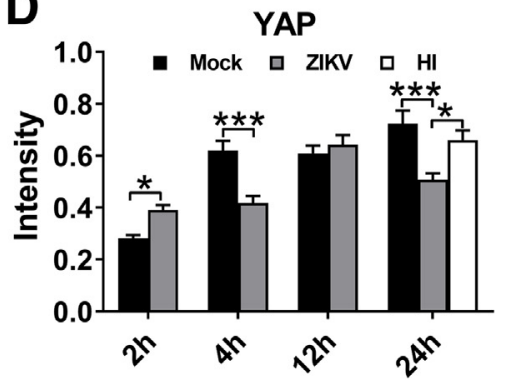

Figure 2 Zika virus (ZIKV) infection affects the expression of Hippo pathway YY1 associated protein 1 (YY1AP1; YAP)/transcriptional co-activator with a PDZ-binding domain (TAZ) factors in human glioblastoma cells. A: U-87 MG human glioblastoma cells support ZIKV replication and produce high titer infectious virus. Graph shows kinetics of ZIKV production by U-87 MG cells. Dotted line represents the assay detection limit. B: Immunohistochemistry images reveal ZIKV infection in U-87 MG cells (green) at 24 hours post-infection (hpi). C: Western blot analysis of YAP/TAZ expression kinetics in ZIKV-infected cells. Longer exposure was required to visualize YAP isoform. Heat-inactivated (HI) ZIKV was included as negative control. D: Graph presents actin-normalized Western blot signal intensity for YAP protein. Representative data from two independent experiments are presented. ${ }^{*} P<0.05,{ }^{* * *} P<0.001(t$-test). Scale bars $=5 \mu \mathrm{m}$ (B). ENV, envelope; PFU, plaque-forming unit.

\section{Zika Viral Infection}

For infection studies, RPE, U-87 MG, and HEK-TIM-1 cells were plated in a 24 -well plate $\left(2.5 \times 10^{4}\right.$ cells/well $)$. After 24 hours, ZIKV inoculum (live or heat inactivated), with a multiplicity of infection of 1 , was prepared using the base media and added to each well $(200 \mu \mathrm{L})$. After 2 to 4 hours of incubation at $37^{\circ} \mathrm{C}$ with $5 \% \mathrm{CO}_{2}$, the base medium was replaced with complete medium ( $1 \mathrm{~mL}$ per well). The uninfected (mock control) wells received complete media without virus inoculum. For energy stress experiment, 2deoxy glucose (2-DG) was added ( $25 \mathrm{mmol} / \mathrm{L}$ solution) to HEK-TIM-1 cells. Cell lysates for protein and RNA were harvested at desired time points for downstream analyses.

\section{Quantification of ZIKV by Plaque Assay}

Vero cells seeded in 12-well plates were used for measuring ZIKV titer of serially diluted cell culture supernatants or serum. Virus inoculum was added to Vero cells and incubated at $37^{\circ} \mathrm{C}$ for 4 hours. Subsequently, complete medium was added. Two days after the infection, viral plaques were counted using inverted phase-contrast microscope (Leica, Buffalo Grove, IL), as previously described. ${ }^{35}$

\section{Mouse Experiment}

This study used 4- to 6-week-old Ifnar ${ }^{-/-}$(IFN- $\left.\alpha \beta R-K O\right)$ male mice [Jackson Laboratory, Bar Harbor, ME; Mutant Mouse Resource \& Research Centers (MMRRC) stock number 32045-JAX] for infection study. The mouse is the most commonly used small animal model system to study ZIKV pathogenesis mechanism in vivo. It provides the opportunity to study ZIKV-mediated brain disease. A recent study ${ }^{26}$ and preliminary study have demonstrated that inoculation of ZIKV in Ifnarl $1^{-/-}$mouse results in brain infection resembling that seen in human patients. Therefore, these mice continue to be used for the ZIKV infection studies. Mice were housed at UCLA Division of Laboratory Animal Medicine. Under isoflurane anesthesia, Ifnar $1^{-/-}$male mice were injected with phosphate-buffered saline (PBS; $n=6$ mice) or PRVABC59 Zika virus $\left(1 \times 10^{6}\right.$ plaque-forming units/mouse in a $40-\mu \mathrm{L}$ volume; $n=6$ mice) in the hind limb region by s.c. route. Blood and tissues were harvested at 7 days post infection (dpi).

On the basis of a previous publication, ${ }^{11} 4.0 \pm 0.5(\log 10$ scale) focus forming units $(\mathrm{FFU}) / \mathrm{mL}$ were estimated in the serum from ZIKV-infected Ifnar ${ }^{-/-}$mice. With a sample size of six animals per group, this study will thus have $80 \%$ statistical power to detect a change between group means of $\geq 1.0(\log 10$ scale). Animals were not excluded from analysis, and the 
only inclusion criterion required was 4- to 6-week-old Ifnarl ${ }^{-/}$

mice. Animals were randomly selected and placed in two groups, PBS control and ZIKV infection groups. Investigators (A.K. and V.A.) were blinded during the acquisition and analysis of images from stained sections of mouse eye and brain.

\section{Immunohistochemistry}

Brain and eye tissues were fixed in $4 \%$ paraformaldehyde for 1 hour and transferred to PBS solution. Tissues were then incubated in 10\% and 20\% sucrose for an hour each and $30 \%$ sucrose overnight. Tissue was then embedded in optimal cutting temperature compound (Fisher Healthcare, Waltham, MA) and stored overnight at $-80{ }^{\circ} \mathrm{C}$. Leica cryostat microtome was used for sectioning tissues $(6 \mu \mathrm{m}$ thick), and the tissue slices were mounted on Super Frost microscope slides (VWR, Aurora, CO). To remove optimal cutting temperature, slides were incubated in $1 \times$ PBS for 10 minutes. Sections were washed three times with $1 \times$ PBS and permeabilized for 1 hour at room temperature using blocking buffer $(0.3 \%$ Triton X-100, $2 \%$ bovine serum albumin, 5\% goat serum, and 5\% donkey serum in $1 \times$ PBS). For immunostaining, sections were submerged in each primary antibody (Table 1) and incubated overnight at $4^{\circ} \mathrm{C}$. Subsequently, the sections were washed three times with $1 \times$ PBS and incubated 1 hour at room temperature with respective secondary antibody (Table 1). Nucleus was visualized by DAPI (Life Technologies) in blocking buffer at a dilution of 1:5000. Images were acquired using ZEISS LSM 700 confocal microscopes (ZEISS, Jena, Germany) and Zeiss Zen Imaging Software version 2.3 available at the UCLA Eli and Edythe Broad Center of Regenerative Medicine and Stem Cell Research Microscopy Core at Center for Health Sciences Building.

\section{Western Blot Analysis}

Cell samples were lysed with lysis buffer containing 50 $\mathrm{mmol} / \mathrm{L}$ Tris ( $\mathrm{pH} 7.4), 0.25 \%$ sodium deoxycholate, $1 \%$ $\mathrm{NP}-40,1 \mathrm{mmol} / \mathrm{L}$ EDTA, $150 \mathrm{mmol} / \mathrm{L} \mathrm{NaCl}, 1 \mathrm{mmol} / \mathrm{L}$ $\mathrm{Na}_{3} \mathrm{VO}_{4}, 20 \mathrm{mmol} / \mathrm{L} \mathrm{NaF}, 1 \mathrm{mmol} / \mathrm{L}$ phenylmethylsulfonyl fluoride, $2 \mathrm{mg} \mathrm{mL}^{-1}$ aprotinin, $0.7 \mathrm{mg} \mathrm{mL}^{-1}$ pepstatin, and $2 \mathrm{mg} \mathrm{mL}^{-1}$ leupeptin or Laemmli Sample Buffer (Bio-Rad, Hercules, CA). SDS-PAGE with $10 \%$ precast gradient gels (Bio-Rad) was used for resolving protein samples. The Trans-Blot turbo transfer system (Bio-Rad) was used for transferring proteins to a $0.2-\mu \mathrm{m}$ polyvinylidene difluoride membrane. Then, the membranes were incubated with 5\% skim milk and $0.1 \%$ Tween-20 in tris-buffered saline at room temperature for 1 hour. After the blocking step, the membranes were incubated with appropriate primary antibodies (Table 1), then secondary antibodies, and detected by SuperSignal West Pico Chemiluminescent Substrate kit (Thermo Scientific, Waltham, MA).
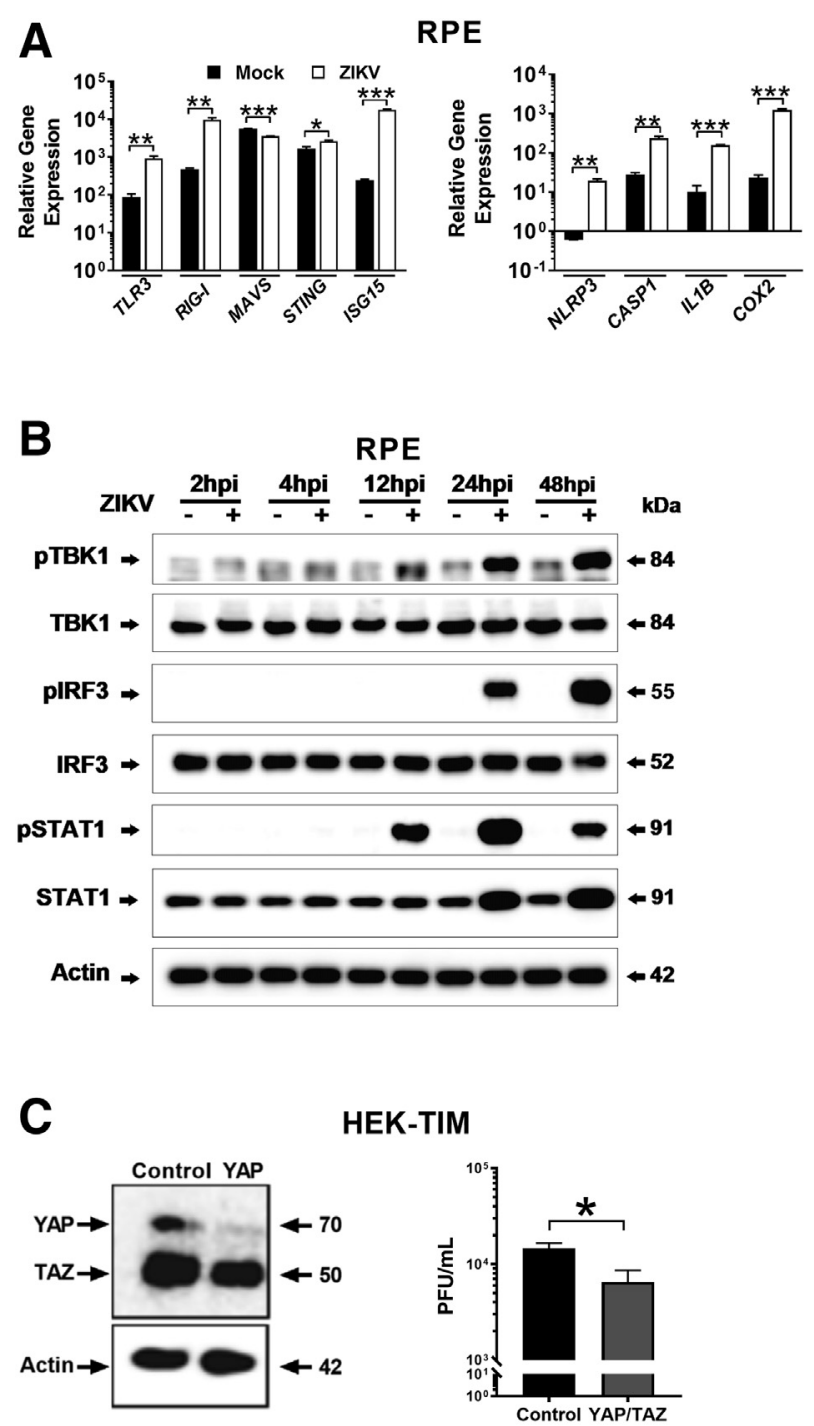

\section{HEK-TIM}

Figure 3 Zika virus (ZIVK) infection modulates inflammatory and immune pathways in retinal pigment epithelial (RPE) cells. YY1 associated protein 1 (YY1AP1; YAP)/transcriptional co-activator with a PDZ-binding domain (TAZ) factors are important for ZIKV replication. A: RNA-sequencing analysis shows genes of innate immune and inflammatory pathways induced on ZIKV infection of RPE cells at 72 hours post-infection (hpi). B: Western blot analysis of innate immune pathway factors activated during ZIKV infection in RPE. C: Western blot of shRNA-mediated knockdown of YAP/TAZ in HEK-TIM-1 cells. Graph depicts ZIKV titer yielded by each shRNA transfected cells at $24 \mathrm{hpi} .{ }^{*} P<0.05,{ }^{* *} P<0.01$, and $* * * P<0.001$ ( $t$-test). PFU, plaque-forming unit; pIRF3, phosphorylated IRF3; pSTAT1, phosphorylated STAT1; pTBK1, phosphorylated TBK1.

\section{Image Analysis/Quantification}

The confocal images were obtained using the Zeiss LSM 700 Confocal Microscopy with the Zeiss Zen Imaging Software version 2.3 with maximum intensity projection feature. By a double-blinded approach (S.W.F. and V.A.), ImageJ's plugin Cell Counter plugin (NIH, Bethesda, MD; https://imagej.nih. gov/ij/plugins/cell-counter.html) was used to count the positively stained cells. Tissue sections from three mice per 

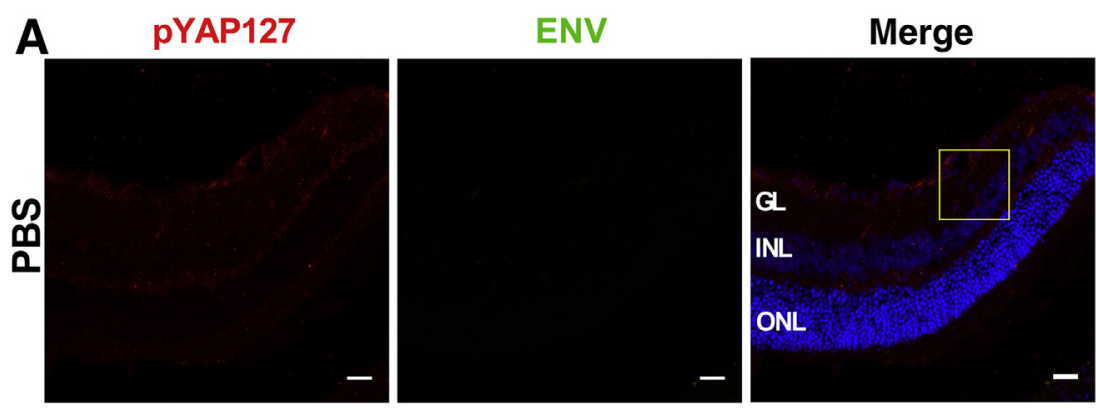

\section{Enlargement}
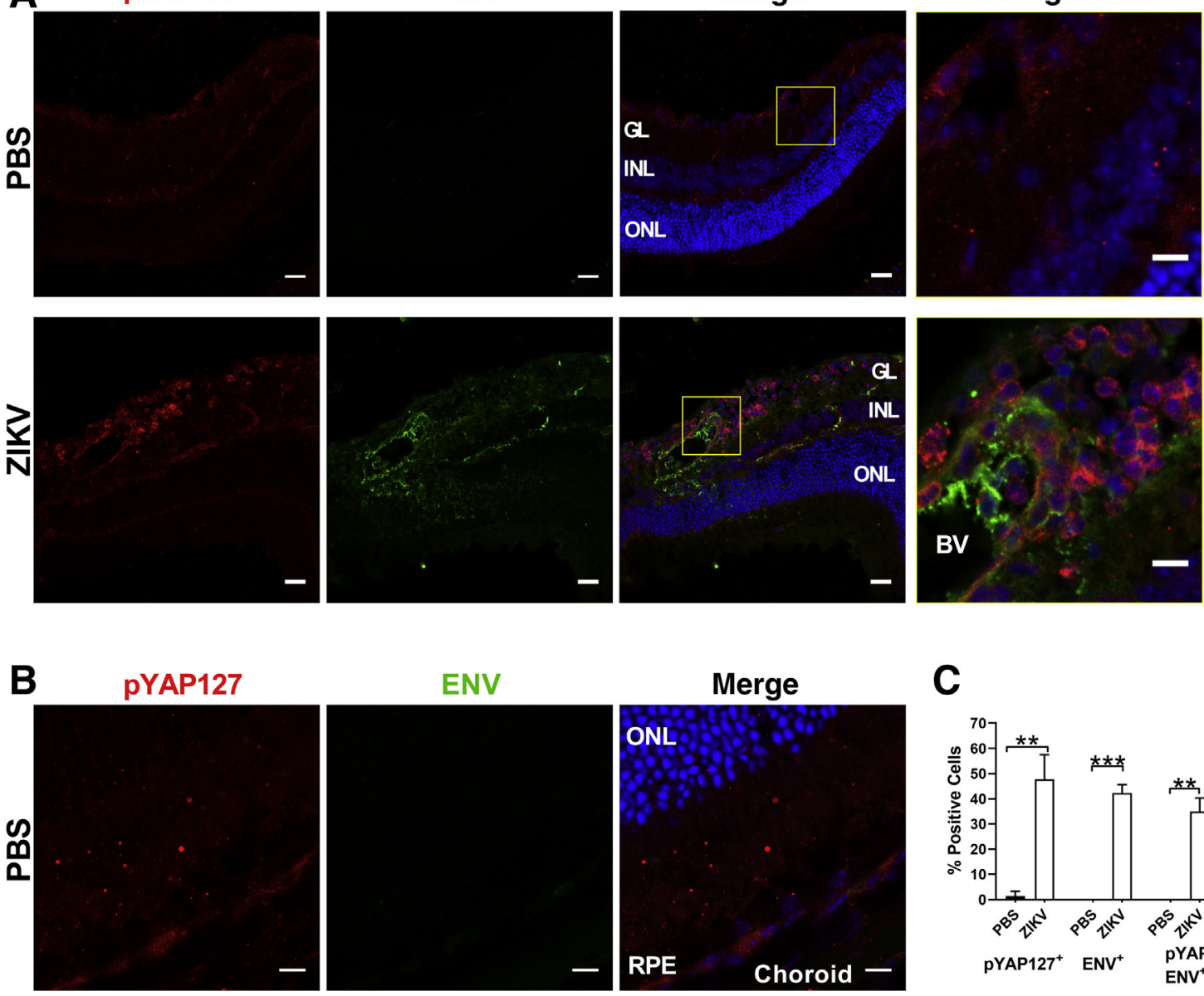

C
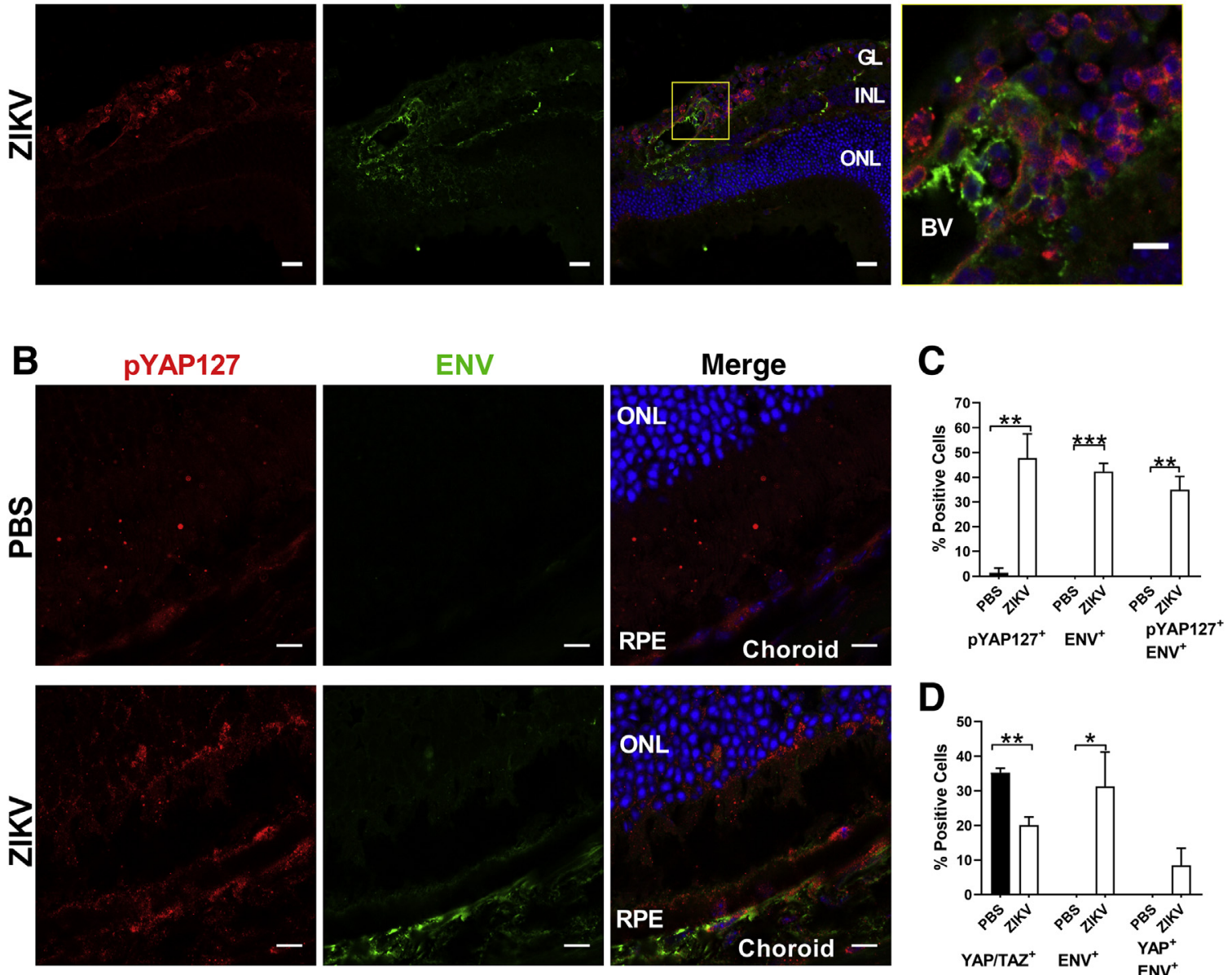

D

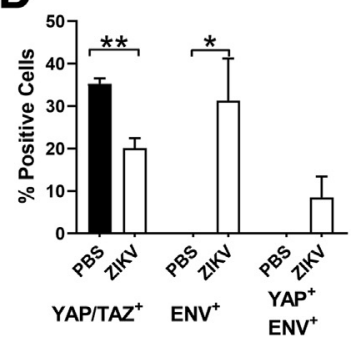

Figure 4 Zika virus (ZIKV) infection induces phosphorylation of YY1 associated protein 1 (Yy1ap1; YAP) protein in retina of Ifnar1 ${ }^{-/-}$mice. A and B: Lowand high-magnification immunohistochemistry images of retina infected with ZIKV are shown. A: Expression of pYAP S127 protein at various layers of retina along with ZIKV envelope (ENV) antigen are presented. Boxed areas are shown at higher magnifications in the enlargments to the right. B: Images of uninfected and infected mice retina, choroidal, and retinal pigment epithelial (RPE) layers, probed for ZIKV and pYAP S127, are shown. C and D: Graphs show quantification of cell populations stained positive for Hippo pathway proteins [pYAP S127 or YAP/transcriptional co-activator with a PDZ-binding domain (TAZ)] and ZIKV ENV in retina. Sections from three mice per group were analyzed with approximately 150 cells counted. Representative data from two independent mice experiments are shown. ${ }^{*} P<0.05,{ }^{*} P<0.01$, and ${ }^{* *} P<0.001$ (t-test). Scale bars: $25 \mu \mathrm{m}(\mathbf{A}$, main images); $5 \mu \mathrm{m}(\mathbf{A}$, enlargements); $10 \mu \mathrm{m}$ (B). BV, blood vessel; GL, ganglion cell layer; INL, inner nuclear layer; ONL, outer nuclear layer; PBS, phosphate-buffered saline.

group were used, and three to four independent images per mouse were analyzed. Confocal images acquired at $63 \times$ objective were used for all the analyses. Total number of cells in each image was obtained by manually counting all the DAPI-stained nuclei using ImageJ version 1.8.0. Total cell count was then used for normalization and to calculate the percentage of individual marker positive cell populations in respective images. The mean percentage of positively stained cells from three to four independent images (approximately 150 cells total) was quantified and presented as graph format.

\section{Caspase 3/7 Assay to Measure Apoptosis}

Caspase-Glo 3/7 Assay was performed as per the manufacturer's protocol (Promega, San Luis Obispo, CA). At 48 hours post-infection (hpi), uninfected and ZIKV-infected RPE cells 
were incubated with the proluminescent caspase- $3 / 7$ substrate at room temperature for 1 hour. Next, $100 \mu \mathrm{L}$ of lysate mix was transferred to a white 96-well microtiter plate, and the luminescence signal was acquired using a luminometer (Glomax Microplate Luminometer; Promega).

\section{RNA Library Preparation and Sequencing}

RPE cells infected with ZIKV were used for RNA sequencing analysis. ${ }^{49} \mathrm{ZIKV}$ at a multiplicity of infection of 1 was used for infection studies. RPE cell lysates were collected at 2 and 3 dpi. For each time point, ZIKV infections were performed in biological triplicates. The uninfected controls (mock infected) were performed in biological quadruplicates and subsequently pooled in duplicates for downstream RNA sequencing analyses. RNeasy Mini Kit (Qiagen, Hilden, Germany) was used for isolating total RNA from the cells. To remove residual carryover DNA, on column RNase-free DNase treatment was performed. Cedars-Sinai Genomics Core was used for library preparation and RNA sequencing analyses. RNA samples were evaluated for quality and concentration using spectrophotometer (NanoDrop 8000; Thermo Scientific). Illumina TruSeq RNA Sample Preparation Kit version 2 (Illumina, San Diego, CA) was used for constructing RNA sequencing libraries, as per manufacturer's instructions. Briefly, total RNA, isolated from uninfected and ZIKVinfected cells having RNA integrity scores of $\geq 9$ (Agilent Bioanalyzer RNA 6000 Nano kit; Agilent, Santa Clara, CA), was used for library preparation. For each sample, the poly(A)+ RNA was enriched using oligo-dT attached magnetic beads and subsequently fragmented and primed for cDNA synthesis. The cDNA was ligated with RNA adapters and barcodes to allow for clonal amplification and multiplexing. Sequencing was performed on an Illumina NextSeq 500 machine using 75-bp single-end sequencing kit. An average read depth of 28 million reads with a minimum number of 16 million reads was obtained for each sample. The sequencing data were submitted to the Gene Expression Omnibus database (https://www.ncbi.nlm.nih. gov/geo; accession number GSE83900).

\section{Analysis of RNA-Sequencing Data}

Raw reads generated from RNA-sequencing experiment were aligned to the human reference genome. STAR software version $2.5^{50}$ was used for alignment with a custom human GRCh38 transcriptome reference that was downloaded from GENCODE (http://www.gencodegenes.org, last accessed January 24, 2017). This reference contained human GENCODE version 23 annotation for all proteincoding as well as protein noncoding RNA genes. A modified trimmed mean of the M-values normalization method was used to normalize expression counts for each gene in all the experimental samples. DESeq2 Bioconductor package version 1.10.1 in $\mathrm{R}$ version 3.2.2 $2^{51}$ was used for performing unsupervised principal component analysis. ${ }^{52}$ Each gene in the samples was fitted into a negative binomial generalized linear model. The differential expression of genes between two sample groups was evaluated by Wald test using DESeq2. To adjust for multiple hypothesis testing, the Benjamini and Hochberg procedure ${ }^{53}$ was employed. To select differentially expressed gene candidates, a false discovery rate of less than the false discovery rate $P<0.05$ was used. The shinyheatmap web interface ${ }^{54}$ was used for the preparation of heat maps. ${ }^{54}$

\section{Statistical Analysis}

All statistical testing was performed at the two-sided $\alpha$ level of 0.05 . To test statistical significance, unpaired $t$-test was used to compare two groups (uninfected versus infected). GraphPad Prism software version 8.1.2 (GraphPad Software, San Diego, CA) was used.

\section{Results}

\section{Hippo Signaling Pathway Is Dysregulated in ZIKV-Infected Cells}

To first understand the molecular perturbations caused by ZIKV infection on human RPE cells, transcriptome analysis ${ }^{49}$ was performed focusing on Hippo and antiviral pathways. The data indicate that many genes in the Hippo signaling pathway at 48 hpi (11 genes) and 72 hpi (21 genes) were differentially regulated (Figure 1A). Specifically, the downstream Hippo pathway transcription coactivators, such as TAZ/WWTRl and TEAD family, were significantly down-regulated $(P<0.05)$ in ZIKV-infected RPE cells. Given that there are changes in the mRNA levels of Hippo pathway related genes, the next approach was to investigate how ZIKV infection affects Hippo pathway at the post-translational level. Before proceeding with the downstream cell-based analysis, we took into consideration that Hippo signaling pathways are dysregulated in cancer cell lines. ${ }^{55,56}$ To minimize the intrinsic effect of Hippo dysregulation, human primary RPE cells were first used and results were verified in immortalized human embryonic kidney cells stably expressing ZIKV entry receptor TIM-1 (HEK-TIM-1) cell line. Interestingly, infected RPEs at 2 hpi had significantly increased YAP protein levels. At 4 hpi, the level of phosphorylated YAP (pYAP) Ser397 form, a signal for YAP degradation, was increased (Figure 1, B and C). The upstream MST1 kinase protein level in ZIKV-infected RPE cells was relatively stable compared with that in uninfected cells, whereas the LATS1 kinase level was reduced (Supplemental Figure S1A). The ZIKV replication was confirmed by detecting the ZIKV non-structural protein 1 (NS1) in infected cells. The result shows that YAP degradation becomes significant as NS1 protein level accumulates to a high level starting at $24 \mathrm{hpi}$ in both cell types. The RPE cells produced high titer infectious 
A

PBS
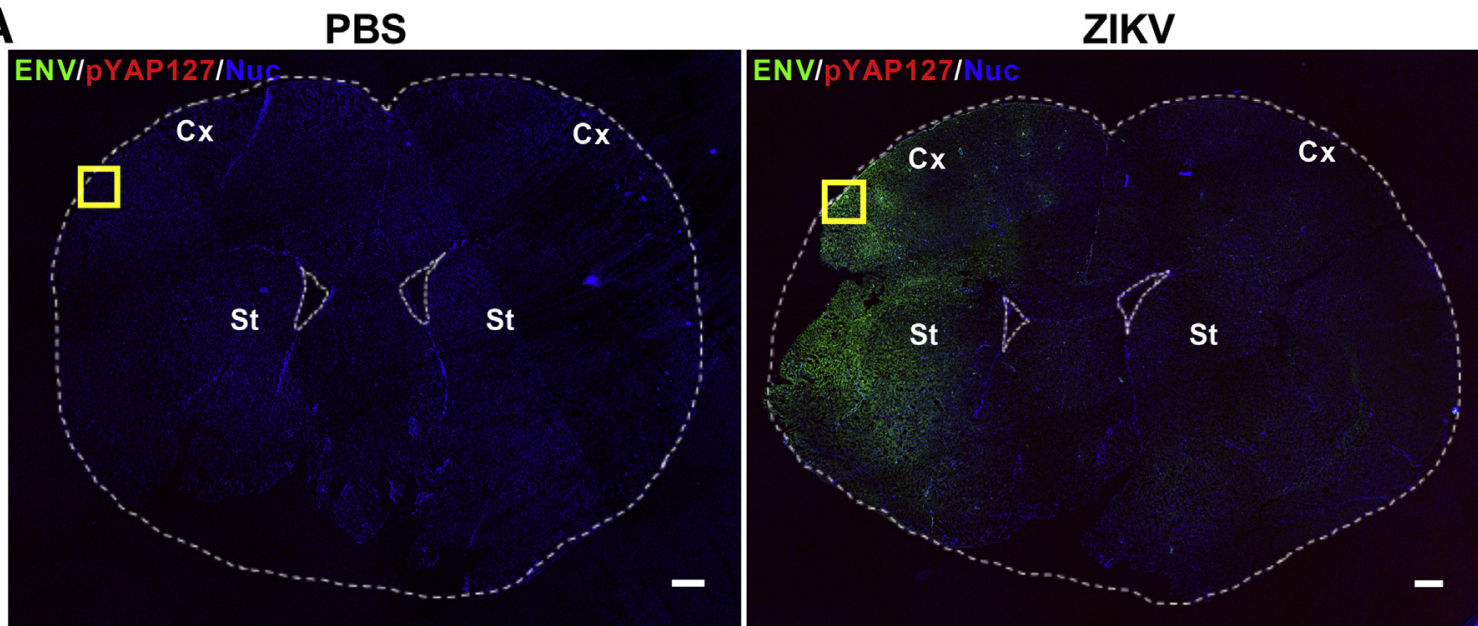

B

pYAP127

ENV

Merge

Enlargement
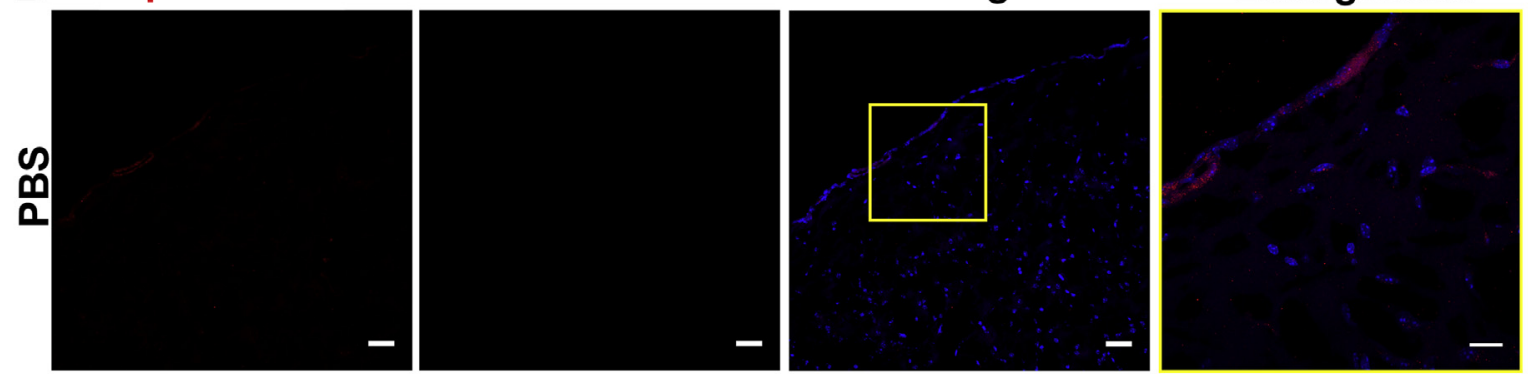

$\frac{Z}{N}$
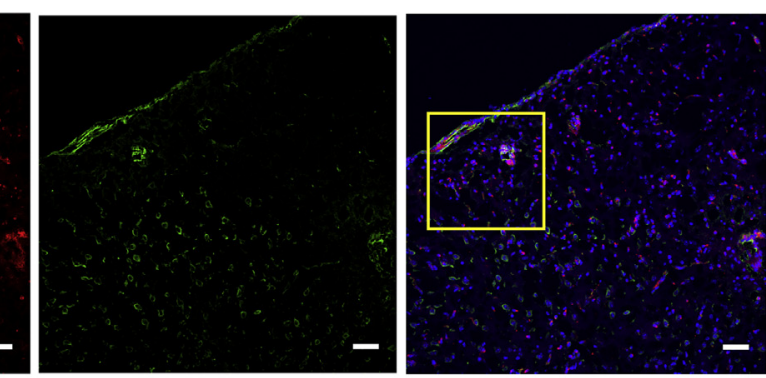

C

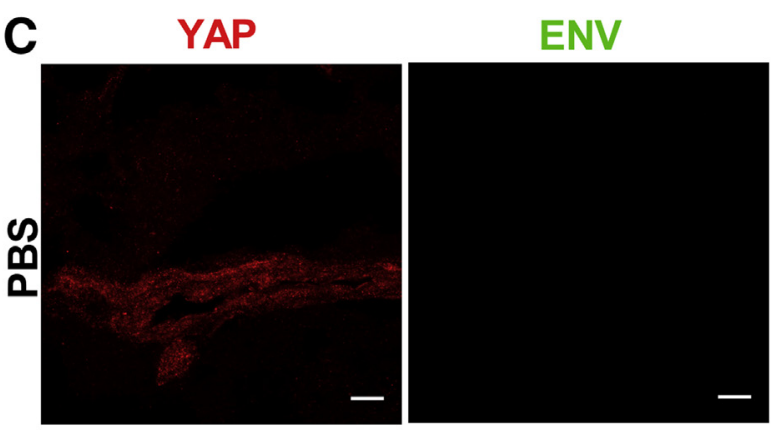

Merge
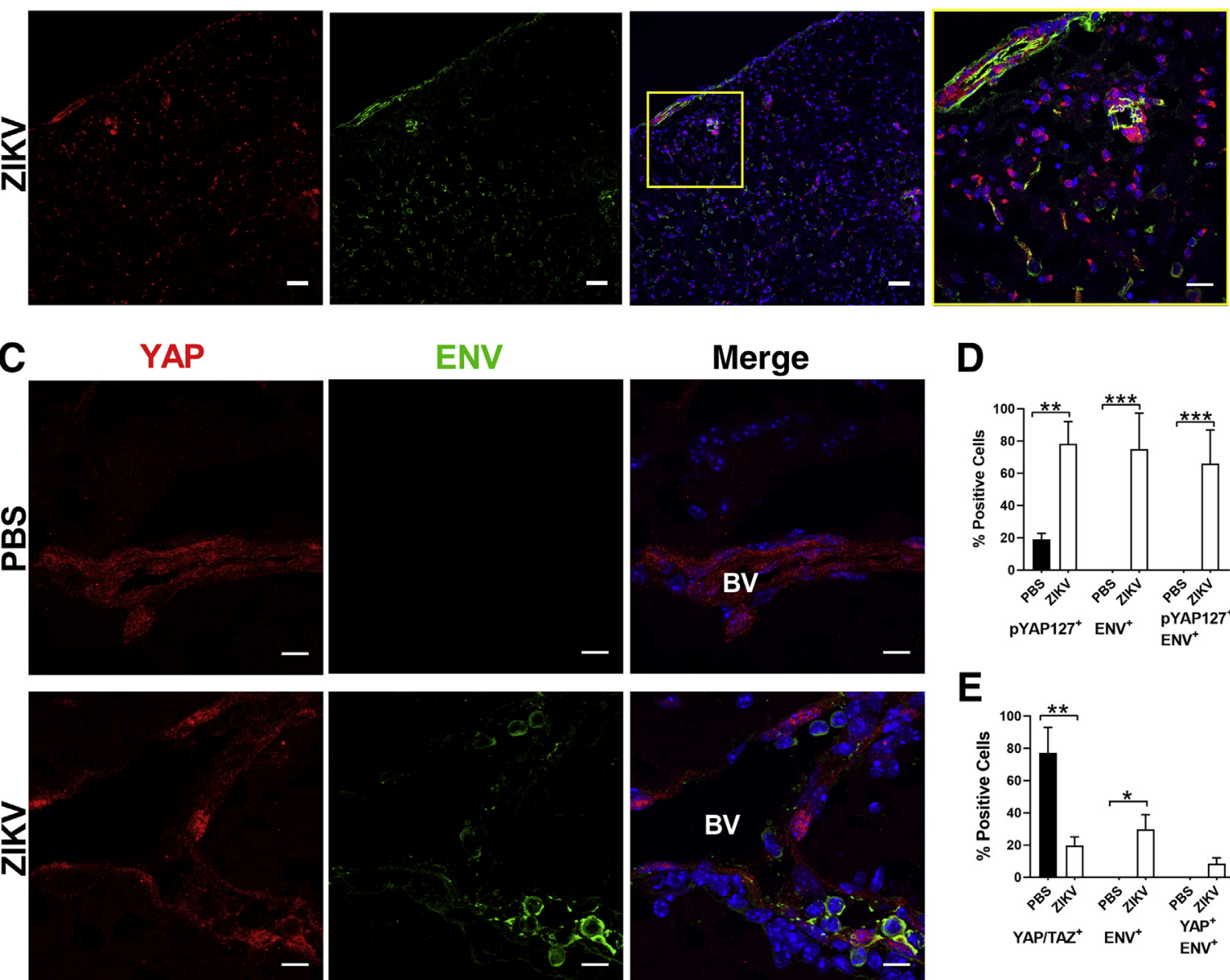

D

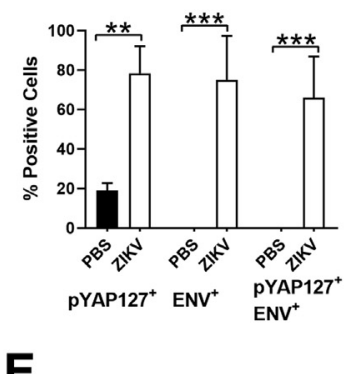

E

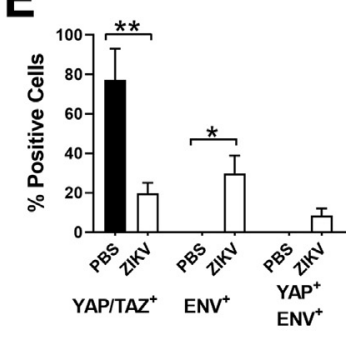

BV

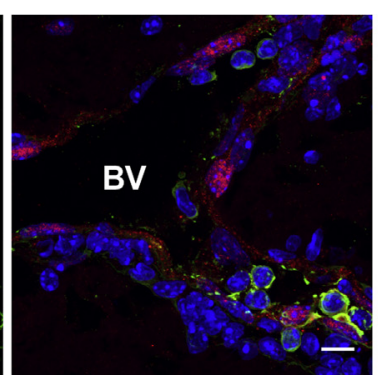


ZIKV at various time points after infection (Figure 1D). More important, RPE cells inoculated with heat-inactivated ZIKV did not have YAP degradation, which suggests that active ZIKV replication is required for Hippo pathway induction. Immunocytochemistry analysis further confirmed the reduction in YAP/TAZ protein levels in infected RPE and HEK-TIM-1 cells (Figure 1E and Supplemental Figure S1B). During fetal infection, ZIKV affects glial progenitors, resulting in microcephaly and associated brain anomalies. This study evaluated the effect of ZIKV infection on Hippo pathway using human glioblastoma cells (Figure 2). These U-87 MG cells have efficiently supported ZIKV infection (Figure 2, A and B), resulting in YAP/TAZ degradation by 24 hpi (Figure 2, C and D). The transformed cell lines, U-87 MG and HEK293, have been passaged in culture for decades, resulting in accumulation of chromosomal abnormalities. Nevertheless, both the HEK-TIM-1 and U-87 MG cell lines, which are highly permissive for ZIKV infection, supported the key findings seen in RPE, demonstrating that dysregulation of the Hippo pathway is not confined to RPE cell type (Figure 1 and Supplemental Figure S1).

Subsequently, the activation of inflammatory and innate antiviral signaling pathways in ZIKV-infected RPE cells were examined (Figure 3, A and B). A time-dependent increase in phosphorylation of key signaling factors TBK1 and IFR3 proteins in ZIKV-infected cells was noted (Figure 3B). Moreover, phosphorylation of STAT1 was observed, indicating that the infection activated the type I and III interferon-mediated innate defense mechanism. In addition, transcriptomic data have revealed the upregulation of TLR3, RIG-I (DDX58), STING, and ISGI5 as well as inflammasome factors $N L R P 3, C A S P 1, I L 1 B$, and COX2 (Figure 3A). YAP/TAZ degradation during ZIKV infection (12 to $48 \mathrm{hpi}$ ) has coincided with the activation of innate immune pathway (Figure 1B). Previous reports have shown that the YAP is an inhibitor of TBK1 and IRF3, and the depletion of YAP can relieve this inhibition. ${ }^{42}$

Moreover, it was observed that ZIKV infection induced caspase 3/7-dependent programmed cell death in RPE cells at 2 dpi (Supplemental Figure S1C). Because of the reduction in total YAP/TAZ level during ZIKV infection, the biological significance of this observation was investigated using shRNA-mediated gene silencing approach. The HEK-TIM-1 cells were transfected with either control shRNA or YAP/TAZ shRNA construct. At 48 hours after transfection, the cells were infected with ZIKV, and
24 hours later, the culture supernatants were collected for measuring viral titer in Vero cells by plaque assay. These data showed that combined YAP/TAZ knockdown resulted in partial reduction of ZIKV replication in HEK-TIM-1 cells (Figure 3C). This result indicates a direct role of Hippo signaling on regulating ZIKV replication. The reduction in YAP/TAZ level observed in infected cells might result from the host cellular response to limit ZIKV replication.

\section{Hippo Signaling Pathway Is Activated by ZIKV in Infected Retina and Brain}

To understand the impact of ZIKV infection on Hippo pathway in vivo, a well-established Ifnarl $^{-1-}$ knockout mouse model was used. The ZIKV-infected mice had a high level of viremia at $7 \mathrm{dpi}$ (Supplemental Figure S2A). Moreover, during the acute phase of infection, these mice exhibited signs of neurologic disease, including posterior paralysis (Supplemental Figure S2B). Using this model, a detailed examination of the molecular and cellular changes in harvested eye and brain tissues was performed at $7 \mathrm{dpi}$ after ZIKV infection. An immunohistochemistry approach was utilized to detect and quantify ZIKV antigen and Hippo pathway YAP/TAZ and pYAP S127 proteins in cells (Figure 4, Supplemental Figure S2, C and D, and Supplemental Figure S3). Ocular sections from three mice per group (three to four images per mouse) were analyzed with approximately 150 cells counted. Mean percentages of positive cells are shown in the graphs. Consistent with in vitro data, YAP/TAZ protein level was reduced in ZIKVinfected retina (Figure 4D and Supplemental Figure S3). A significant increase in YAP Ser127 phosphorylated form in various cell layers of retina, such as ganglion cell outer nuclear and RPE layers in the infected mice was noted (Figure 4, A-C). ZIKV infection was observed, detected by viral envelope positive cells, in the choroidal endothelium, indicating virus replication in the blood-retinal barrier (Figure 4B). YAP Ser127 phosphorylation will exclude YAP from nucleus, thus preventing its cotranscriptional activity. These data provide evidence that host response might favor nucleus exclusion and degradation of YAP/ TAZ to restrict ZIKV infection.

Because ZIKV causes encephalitis in adults, a careful evaluation of infected mouse brain was performed to understand the role of Hippo pathway during acute neuroinflammation. In normal uninfected brain, YAP/TAZ

\footnotetext{
Figure 5 Zika virus (ZIKV) infection activates Hippo signaling pathway in brain of Ifnar1 ${ }^{-1-}$ mice. A: Low-magnification immunohistochemistry images of uninfected and ZIKV-infected brains are shown. The $5 \times 5$ tiles of $5 \times$ objective confocal images were stitched to obtain the full image. Dashed circles show the outline of the brain cross-sections. B: High-magnification images of the highlighted cortical regions (boxed areas) are provided. B: Images show induction of S127 phosphorylated YY1 associated protein 1 (Yy1ap1; YAP) protein in ZIKV-infected brain. Boxed areas are shown at higher magnification in the enlargements to the right. C: Images of brain sections immunostained for YAP and envelope (ENV) proteins are shown. D and E: Graphs show quantification of cells positive for Hippo pathway proteins and ZIKV ENV in brain. Sections from three mice per group were analyzed, with approximately 150 cells counted. Representative data from two independent mice experiments are shown. ${ }^{*} P<0.05,{ }^{* *} P<0.01$, and ${ }^{* * *} P<0.001$ ( $t$-test). Scale bars: $200 \mu \mathrm{m}(\mathbf{A}) ; 25 \mu \mathrm{m}(\mathbf{B}$, main images); $10 \mu \mathrm{m}$ (B, enlargements, and C). BV, blood vessel; Cx, cortex; PBS, phosphate-buffered saline; St, striatum.
} 
A
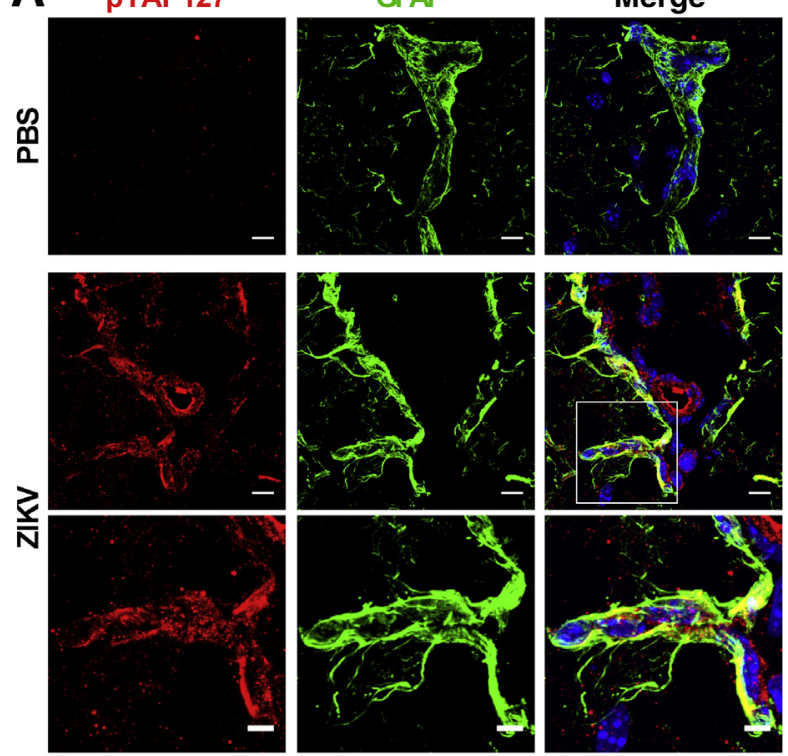

C
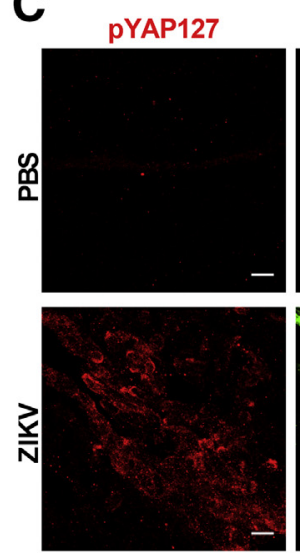

Occludin

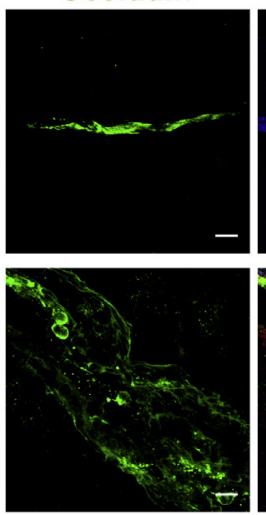

Merge

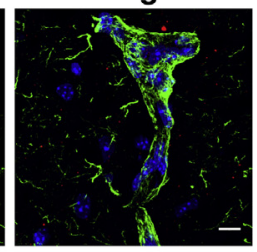

Merge

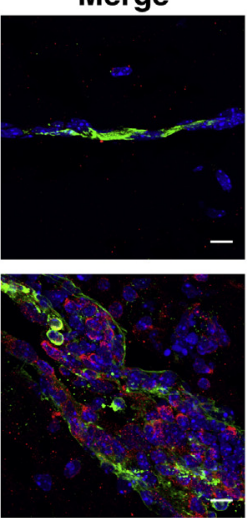

B

pYAP127
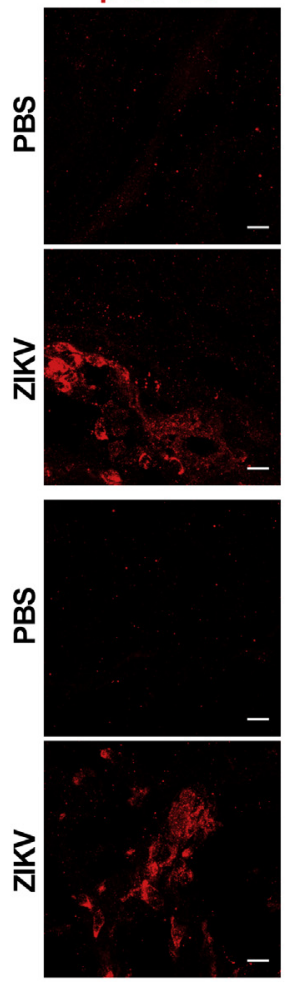

D

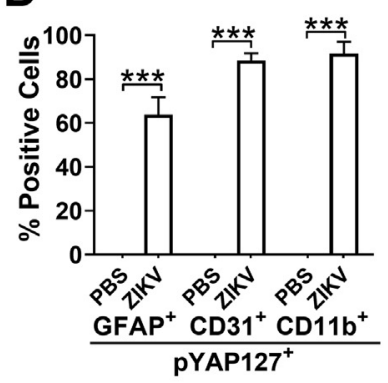

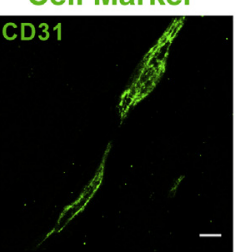
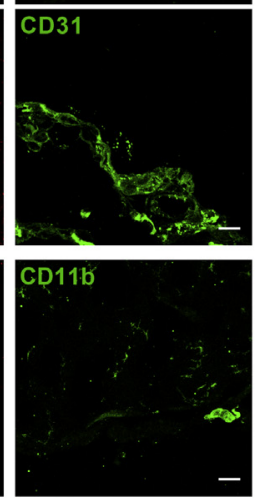

Merge
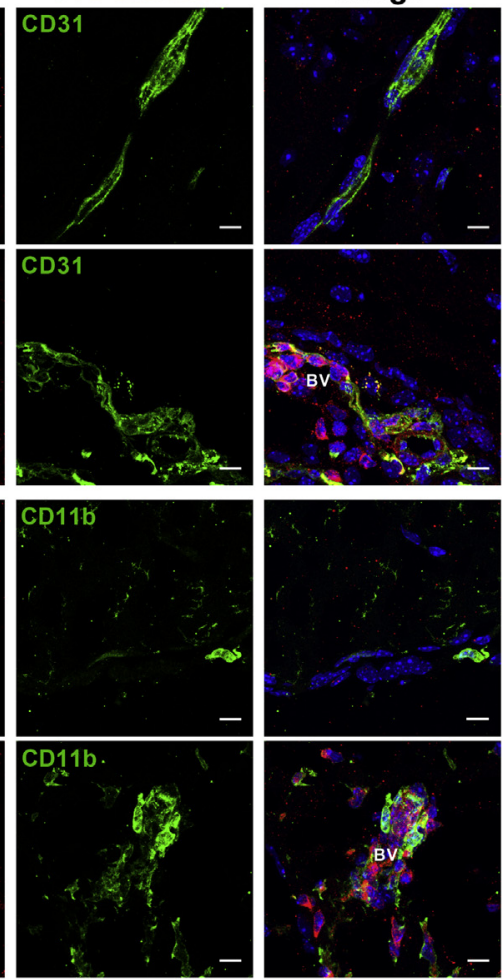

E

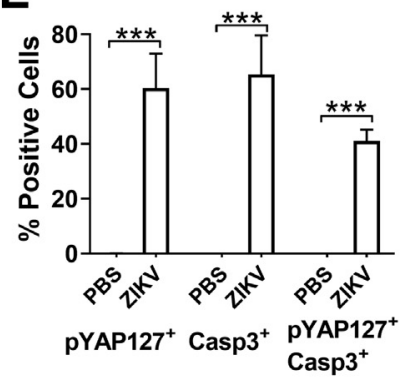

Figure 6 Activation of Hippo pathway in the components of blood-brain barrier during acute Zika virus (ZIKV) neuroinflammation. A: Immunohistochemistry analysis of astrocytes [glial fibrillary acidic protein positive $\left(\mathrm{GFAP}^{+}\right)$] during ZIKV infection. Boxed area is shown at higher magnification in the bottom row. B: Analyses of endothelial cell $\left(\mathrm{CD} 31^{+}\right)$and microglial cell $\left(\mathrm{CD} 11 \mathrm{~b}^{+}\right)$populations in ZIKV-infected brain. C: Images show the alteration of brain vasculature tight junction (occludin ${ }^{+}$) continuity by infiltrating cells and inflammatory condition in infected mouse brain. D: Graph shows percentage of cell population containing phosphorylated form of YY1 associated protein 1 (Yy1ap1; YAP) in ZIKV-infected brain sections. E: Cleaved caspase 3 positive cells quantified in infected brain are shown. Sections from three mice per group were analyzed, with approximately 150 cells counted. ${ }^{* * *} P<0.001$ ( $t$-test). Scale bars: $10 \mu \mathrm{m}$ (A, top and middle row, B, and $\mathbf{C}) ; 5 \mu \mathrm{m}$ (A, bottom row). BV, blood vessel; PBS, phosphate-buffered saline.

protein expression was observed in cells lining the ventricular zone, astrocytes, and the vascular endothelial cells (Supplemental Figure S2C). Low to below detectable levels of YAP/TAZ were noticed in cortical neurons. ZIKV infection resulted in acute inflammatory changes, including the perivascular leakage of inflammatory cells into the brain parenchyma (Figure $5, \mathrm{~A}-\mathrm{C}$ ). The changes in YAP/TAZ and pYAP S127 levels in the brain were quantified by counting the cells expressing these proteins in mice that were mock or ZIKV infected (Figure 5, D and E). Consistent with the in vitro and retinal findings, the pYAP S127 level in mouse brain is significantly increased on ZIKV infection (Figure 5, B and D), denoting the activation of Hippo signaling pathway. Conversely, the YAP/TAZ expressing cells were reduced in the infected mouse brain (Figure 5, C and E). These observations indicate that acute oculoneuroinflammatory condition favored the inactivation of YAP/TAZ by its 

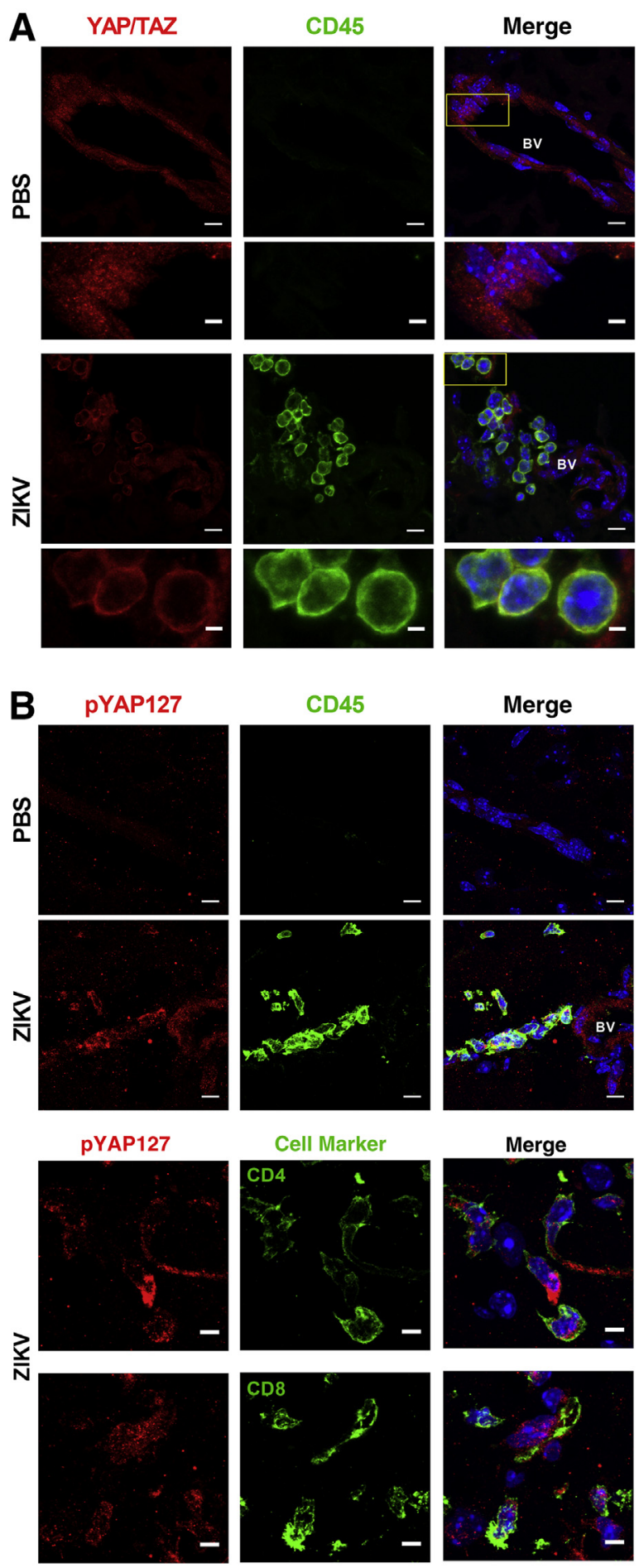

\begin{abstract}
Cell Marker
\end{abstract}
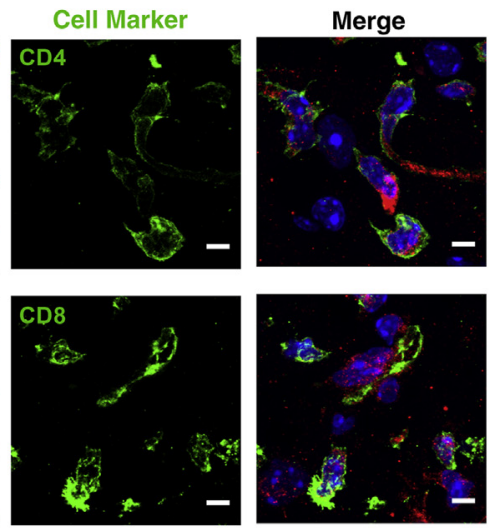

C

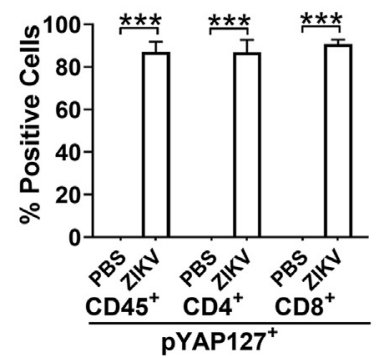

phosphorylation, and further suggest that the Hippo pathway might orchestrate the host-pathogen interaction during retinal and brain inflammation.

Acute ZIKV Neuroinflammation Leads to Activation of Hippo Pathway in the BBB

Given ZIKV is capable to cross the BBB, we specifically focused on the effect of infection on major components of the BBB: astrocytes (glial fibrillary acidic protein positive), endothelial cells $\left(\mathrm{CD} 31^{+}\right)$, and microglia $\left(\mathrm{CD} 11 \mathrm{~b}^{+}\right)$(Figure 6, A-D). ZIKV infection resulted in formation of astrogliosis, microglial nodules, and apoptotic cell death (Figure 6, A, B, and E, and Supplemental Figure S4). In infected mouse brain, the tight junctions of the vasculature could be altered by infiltrating cells and inflammatory condition, as shown by loss of occludin continuity in affected blood vessels by immunostaining (Figure 6C). ZIKV effect on the components of BBB implicates that the brain vascular pericytes could be compromised as well. In addition, ZIKV-infected brain parenchyma and vasculature had high levels of inflammatory $\mathrm{CD} 45^{+}$cells. These cells show YAP/TAZ localization to cytoplasm (Figure 7A), as well as higher level of cytoplasmic pYAP S127, both observations suggesting that the Hippo signaling pathway is activated in response to viral infection (Figure 7B). Additional immune cell $\mathrm{CD}^{+}{ }^{+}$and $\mathrm{CD}^{+}{ }^{+}$T-cell populations showed high expression of pYAP S127 as well (Figure 7, B and C). Taken together, these data reveal that Hippo pathway activation might be important for the host inflammatory and immune cells' activities during viral-mediated neuroinflammation.

\section{Cross Talk between Hippo Signaling and Energy Stress Pathways}

Virus replication exerts huge energy demands on host cells with increased glucose/ATP consumption. The AMP/ATP ratio has been shown to be increased during ZIKV infection. ${ }^{26}$ This energy stress can induce activation of the master energy pathway regulator AMP-activated protein kinase $\alpha$ $(\mathrm{AMPK} \alpha)$ to promote glycolysis and fatty acid oxidation. Although AMPK $\alpha$ activation has been linked to degradation

Figure 7 Hippo signaling is active in infiltrating inflammatory and immune cells during Zika virus (ZIKV) neuroinflammation. A: Infiltration of $\mathrm{CD} 5^{+}$cells from brain vasculature to parenchyma during ZIKV infection is shown. Note that YY1 associated protein 1 (Yy1ap1; YAP)/transcriptional coactivator with a PDZ-binding domain (TAZ) is excluded from nucleus and cytoplasmically localized in the inflammatory $\mathrm{CD} 45^{+}$cells. Boxed areas are shown at higher magnification in the enlargements below. B: Hippo pathway is activated in infiltrating $\mathrm{CD}_{4} 5^{+}, \mathrm{CD}^{+}$, and $\mathrm{CD}^{+}$cells with increased level of pYAP S127. C: Graph presents the percentage of cell population containing pYAP S127 in ZIKV-infected brain sections. Sections from three mice per group were analyzed, with approximately 150 cells counted. ${ }^{* *} P<0.001(t$ test). Scale bars: $10 \mu \mathrm{m}$ (A, main images, and $\mathbf{B}) ; 5 \mu \mathrm{m}$ (A, enlargements). BV, blood vessel; PBS, phosphate-buffered saline. 
A
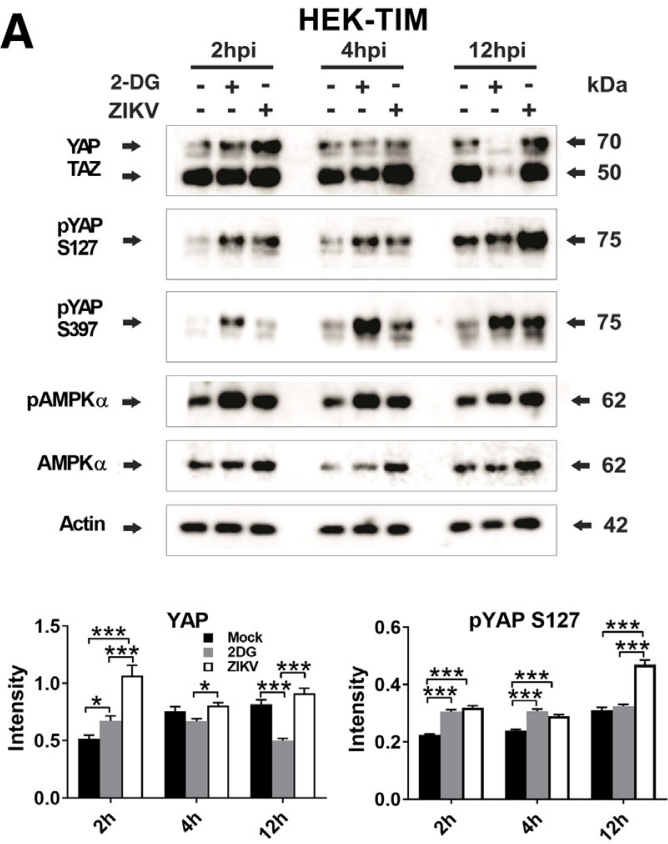

C

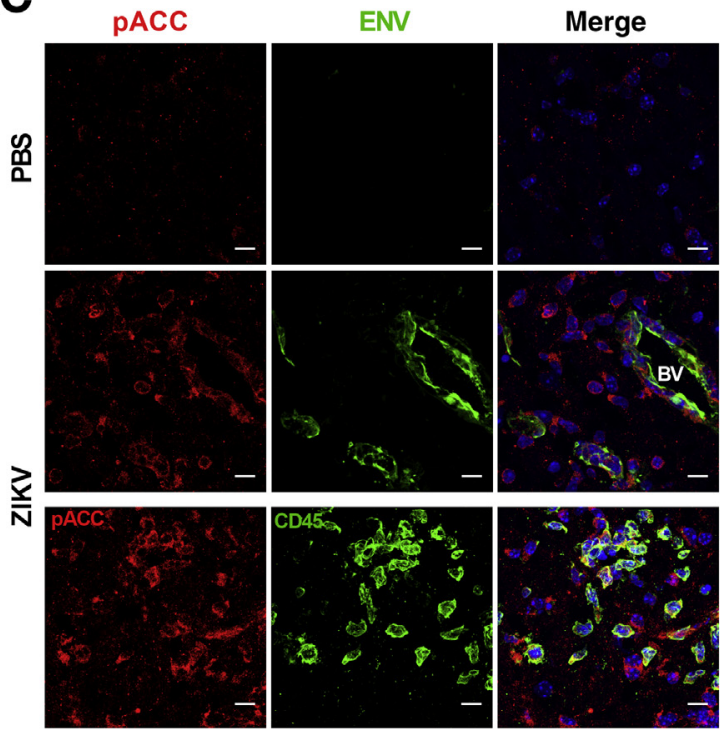

B RPE

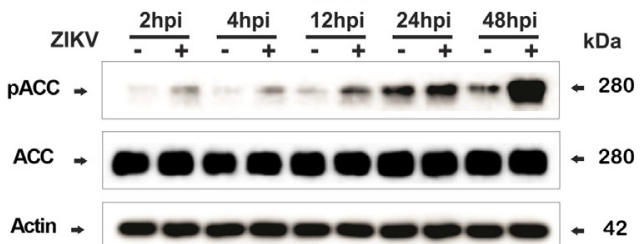

HEK-TIM

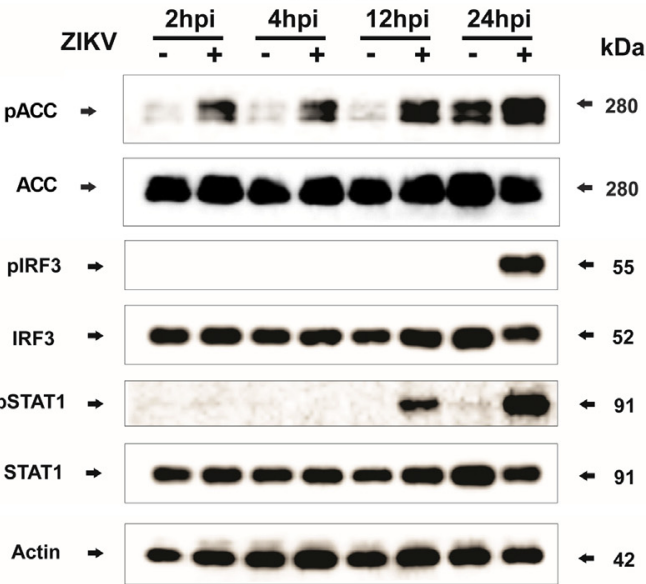

D

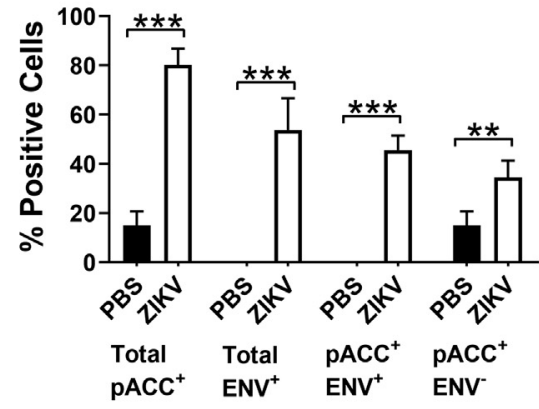

Figure 8 Adenosine monophosphate (AMP)-activated protein kinase (AMPK)-Hippo signaling pathways' cross talk during Zika virus (ZIKV) infection. A: Western blot analysis of HEK-TIM-1 cells treated with energy stress inducer 2-deoxy glucose (2-DG; $25 \mathrm{mmol} / \mathrm{L})$ and ZIKV (multiplicity of infection 1) at various time points. Graphs show actin-normalized Western blot signal intensity for YAP and pYAP S127 proteins. B: Phosphorylation of AMPK $\alpha$ (pAMPK $\alpha$ ) downstream target protein acetyl-CoA carboxylase (ACC) as well as innate immune pathway factors in ZIKV-infected cells are shown. C: Immunohistochemistry images of mice brain sections illustrate phosphorylated form of ACC (pACC) during acute ZIKV neuroinflammation and CD45 ${ }^{+}$leukocytes costained for pACC. D: Graph depicts quantification of pACC positive cells. Sections from three mice per group were analyzed, with approximately 150 cells counted. ${ }^{\star} P<0.05$, ${ }^{*} P<0.01$, and ${ }^{* * *} P<0.001$ (t-test). Scale bars $=10 \mu \mathrm{m}(\mathrm{C})$. BV, blood vessel; ENV, envelope; pIRF3, phosphorylated IRF3; pSTAT1, phosphorylated STAT1.

of YAP/TAZ through LATS1/2-mediated phosphorylation at YAP Ser127, ${ }^{57,58}$ the cross talk between these two pathways during ZIKV infection is not known. Therefore, we set out to elucidate a novel link between AMPK and Hippo pathway during ZIKV infection. 2-DG, a well-known glycolytic inhibitor, and AMPK pathway activator, as a positive control, were used. ${ }^{59}$ The data revealed that both 2-DG treated and ZIKV-infected cells showed timedependent phosphorylation of YAP and AMPK $\alpha$ (Figure 8A). Significant elevation in the phosphorylated YAP S127 form was observed in the 2-DG treated and ZIKV-infected cells. This experiment showed that ZIKV 
infection is mimicking the molecular changes that are observed in cells subjected to 2-DG mediated energy stress. Subsequently, acetyl-CoA carboxylase (ACC), a key downstream target of activated AMPK $\alpha$, was examined. ACC is a multifunctional catalyst that accelerates the conversion of acetyl-CoA to malonyl-CoA for fatty acid synthesis, which is negatively regulated by AMPK-mediated phosphorylation of ACC at serine residue $79 .{ }^{60}$ ACC phosphorylation due to energy stress promotes fatty acid oxidation. Time-dependent increases in phosphorylated ACC form in ZIKV-infected RPE and HEK-TIM-1 cells were observed (Figure 8B). The result suggested that ZIKVinfected cell might activate AMPK $\alpha$ to counteract the energy stress, which could subsequently induce the Hippo signaling pathway. However, to elucidate the direct link between AMPK-Hippo pathway, additional functional studies are required. Similarly, an increase of phosphorylated ACC containing cells in the ZIKV-infected brain was observed (Figure 8, C and D). Both ZIKV-infected brain cells and infiltrating $\mathrm{CD} 45^{+}$inflammatory cells had high level of phosphorylated ACC. This result suggests that the cellular metabolism might be reprogrammed in the inflammatory niche. Our current model, based on this, and previous studies, suggests an interesting hypothesis that in a normal state (Hippo inactive), YAP/TAZ is able to translocate into the nucleus and regulate cell proliferation and survival. During infection, the AMPK, activated by energy stress, can induce the Hippo signaling pathway, resulting in YAP inactivation by phosphorylation. Because YAP is known to negatively regulate TBK1 antiviral pathway, depletion of YAP can promote antiviral response (Figure 9). Hence, this study reveals an intriguing relationship between ZIKV and AMPK-Hippo pathway that might play an important role in host inflammatory and antiviral responses.

\section{Discussion}

The role of the Hippo signaling pathway in organ development and cell differentiation and proliferation is well established. ${ }^{35,61,62}$ Herein, we provide evidence that this pathway has broader biological role during host's inflammatory and immune responses against invading pathogens, specifically the Zika virus, a member of Flaviviridae family. Using a clinically relevant in vitro human fetal RPE cell-based system, we demonstrate that ZIKV directly affects the Hippo signaling pathway (Figure 1 and Supplemental Figure S1). This observation has clear implications during the pathogenesis of congenital ZIKV eye disease in developing fetal retina as the irreversibly injured RPE cells undergo apoptotic cell death. A dysregulated Hippo pathway in the affected retina can further hamper retinal progenitor cell differentiation and retinal organogenesis. The initial up-regulation of the YAP protein that was observed could be because of the virus trying to influence the host machinery to promote favorable cell conditions for its replication (Figure 1). However, in turn, the host could respond to counter ZIKV infection by activating an antiviral response and the Hippo pathway, leading to the degradation of the critical proviral factor YAP/TAZ. Similar observations of YAP depletion were reported during Sendai virus and herpes simplex virus 1 infection in HEK293T cells. ${ }^{42}$ After YAP depletion, there was consequent relief of YAP-mediated inhibition of cellular antiviral pathway factors TBK1 and IRF342. Future studies in cell lines lacking TBK1, IRF3, LATS, or YAP genes can help address the specific relationship between innate immunity and Hippo pathway during ZIKV infection. A recent study demonstrated that ZIKV infection modulated the methylation status of several Hippo pathway genes, such as TAZ and Ras association domain family member 1 (RASSF1), in human neural progenitor cells. ${ }^{63}$
A

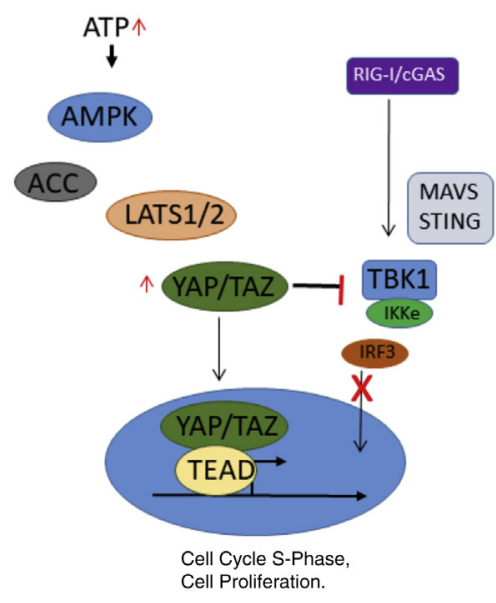

B Infected/Energy Stress State

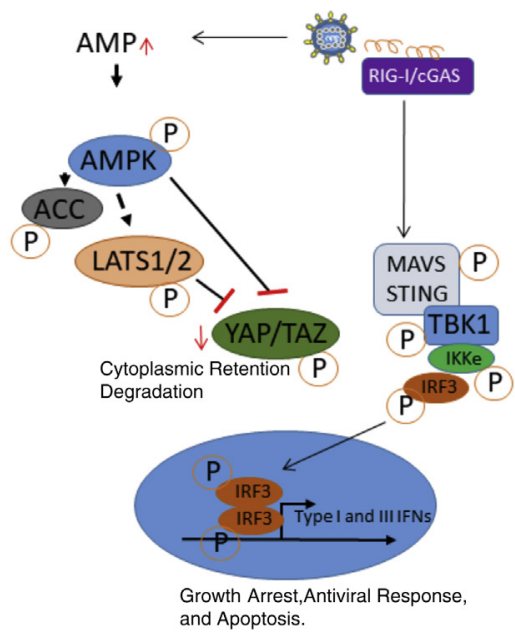

Figure 9 Schematic diagram of our hypothetical model integrating AMP-activated protein kinase (AMPK), Hippo, and TBK1 signaling pathways during normal (A) and ZIKV-infected states (B). ACC, acetyl-CoA carboxylase. AMP, adenosine monophosphate; ATP, adenosine triphosphate; cGAS, cyclic GMP-AMP synthase; IKKe, inhibitor of nuclear factor kappa B kinase subunit epsilon; MAVS, mitochondrial antiviral-ignaling protein; RIG-I, retinoic acidinducible gene I protein; STING, stimulator of interferon response CGAMP interactor 1; TEAD, TEA domain transcription factors; ZIKV, Zika virus. 
The mouse model in this study confirmed that the Hippo pathway is specifically activated during Zika viral infection of the retina and brain (Figure 4 and 5). Because the YAP degradation is observed in Innarl $^{-/-}$mice tissues during infection, the YAP inactivation is regulated upstream of the type I interferon Janus kinase (JAK)-STAT1 signaling pathway. It is well established that wild-type C57BL/6 mice are resistant to flaviviruses, including ZIKV. Therefore, most studies have used immunocompromised Ifnarl $^{-1-}$ mice that are devoid of receptor for both type I and II interferons. However, it is important to acknowledge the limitations of Ifnarl ${ }^{-1-}$ mouse model for ZIKV pathogenesis study. Ifnarl ${ }^{-/-}$mouse lacks interferon $(\alpha$ and $\beta$ ) receptor 1 subunit, which is critical for activation of JAKSTAT1 pathway-mediated innate antiviral response. This defect can lead to increased susceptibility to ZIKV infection with higher mortality and viral load than infected wild-type mouse. Moreover, this model has also been shown to be defective for natural killer cell-mediated antitumor function as well. ${ }^{64}$ However, Ifnarl ${ }^{-/-}$mice have functional type III IFN system as well as B- and T-cell-mediated adaptive immune system.

The Hippo pathway is known to be controlled by mechanical stress, sheer force, and cell-cell contact. ${ }^{37,65}$ High levels of pYAP S127 form in infected and activated endothelial cells in the brain vasculature were observed in this study. In blood vessels, the nature of blood flow (laminar versus turbulent) can have an effect on Hippo signaling, as it has been demonstrated to play a role in the pathogenesis of atherosclerosis. ${ }^{66}$ It is possible that in the inflamed brain vasculature, the tremendous volume of leukocyte infiltration could impede the vascular flow and affect the Hippo pathway. Another interesting observation in our study is that the Hippo pathway is active in astrocytes. Multiple flaviviruses (West Nile virus and Japanese encephalitis virus), including ZIKV, have been shown to infect astrocytes, as it is vital for retention of virus in the central nervous system. ${ }^{67-70}$ Hippo pathway activity in astrocytes can help restrict virus replication and mount inflammatory response. A previous study using Yap $^{\text {nestin }}$ conditional knockout mice shows depletion of YAP led to astroglyosis and astrocyte-driven microglial activation. ${ }^{71}$ This study supports the observation that Hippo pathway activation in astrocytes may contribute to neuroinflammation.

It has been reported that the upstream Hippo pathway mediator MST1 is important for T-cell function. In humans, mutation in MST1 gene has been shown to have an immune deficiency phenotype due to defective $\mathrm{T}$ cells. ${ }^{41,72}$ It was observed that the brain-infiltrating $\mathrm{CD}^{+}$and $\mathrm{CD}^{+} \mathrm{T}$ lymphocytes have activated Hippo pathway, which suggests that YAP/TAZ functional inactivation could be one of the triggers for activating the T-cell-mediated acquired immune response. The infiltrating inflammatory cells were also noticed to have high level of phosphorylated ACC. This interesting observation implies that fatty acid oxidation is also required for infiltrating cells' activity during neuroinflammation.

AMPK pathway has been shown to play an important role in innate immune defense in various infectious and inflammatory conditions. ${ }^{73-75}$ Moreover, AMPK $\alpha$ plays a pivotal role in inhibiting flavivirus (West Nile virus, ZIKV, and Dengue virus). ${ }^{76}$ Recent reports show that AMPK pathway activators 5-aminoimidazole-4-carboxamide ribonucleotide (AICAR) and metformin can inhibit ZIKV replication by modulating the host-cell metabolism. ${ }^{77,78}$ There has been a concerted effort to identify and develop drugs that can modulate the Hippo pathway. Previous studies have shown that HMG-CoA reductase inhibitors, such as statins (cholesterol-lowering drugs), are effective activators of the Hippo pathway. ${ }^{79,80}$ It would be useful to test statins during acute viral neuroinflammation to evaluate a potential neuroprotective effect.

These findings have opened up exciting new areas for further investigation. It is important to identify ZIKVencoded virulence factors that can affect the AMPKHippo signaling pathways, as this will provide functional and mechanistic insight. Mouse genetic models with cellspecific conditional YAP or LATS knockout studies in astrocytes, RPE, and mononuclear cells would be useful to tease apart the complexity of the Hippo pathway role in viral retinitis and encephalitis. In summary, these results indicate that the Hippo signaling pathway plays a fundamental role in regulating immune and inflammatory processes during ZIKV infection. Thus, the Hippo signaling pathway can be a potential therapeutic target for the management of both ocular and neuronal inflammation.

\section{Acknowledgments}

We thank Ken Yamauchi, Ph.D. (Novitch laboratory); the Eli and Edythe Broad Center of Regenerative Medicine and Stem Cell Research Microscopy Core (UCLA); the Translational Pathology Core Laboratory (UCLA); Priya Gyani for technical assistance for Western blot analysis; and Sophia Paiola for assistance with immunohistochemistry.

\section{Author Contributions}

G.G.Jr. and S.P. concieved and designed the study, collected and analyzed data, and wrote the manuscript; S.B., collected and analyzed the data and wrote the manuscript; V.K.R., A.R., K.N.-S., M.M.H.L., S.W.F., and K.M., analyzed the data; A.K., designed experiments and analyzed the data; V.A., concieved and designed the study, provided financial support, analyzed the data, and wrote the manuscript; all authors approved the manuscript. 


\section{Supplemental Data}

Supplemental material for this article can be found at http://doi.org/10.1016/j.ajpath.2019.12.005.

\section{References}

1. Duffy MR, Chen T-H, Hancock WT, Powers AM, Kool JL, Lanciotti RS, Pretrick M, Marfel M, Holzbauer S, Dubray C, Guillaumot L, Griggs A, Bel M, Lambert AJ, Laven J, Kosoy O, Panella A, Biggerstaff BJ, Fischer M, Hayes EB: Zika virus Outbreak on Yap Island, Federated States of Micronesia. N Engl J Med 2009, 360:2536-2543

2. Tsui I, Moreira MEL, Rossetto JD, Vasconcelos Z, Gaw SL, Neves LM, Zin OA, Haefeli L, Silveira Filho JCB, Gomes SC Jr, Adachi K, Pone M, Pone SM, Pereira JP Jr, Belfort $\mathrm{R}$, Arumugaswami V, Brasil P, Nielsen-Saines K, Zin AA: Eye findings in infants with suspected or confirmed antenatal Zika virus exposure. Pediatrics 2018, 142:e20181104

3. Brasil P, Pereira JP Jr, Moreira ME, Ribeiro Nogueira RM, Damasceno L, Wakimoto M, Rabello RS, Valderramos SG, Halai UA, Salles TS, Zin AA, Horovitz D, Daltro P, Boechat M, Raja Gabaglia C, Carvalho de Sequeira P, Pilotto JH, Medialdea-Carrera R, Cotrim da Cunha D, Abreu de Carvalho LM, Pone M, Machado Siqueira A, Calvet GA, Rodrigues Baiao AE, Neves ES, Nassar de Carvalho PR, Hasue RH, Marschik PB, Einspieler C, Janzen C, Cherry JD, Bispo de Filippis AM, Nielsen-Saines K: Zika virus infection in pregnant women in Rio de Janeiro. N Engl J Med 2016, 375:2321-2334

4. Ventura CV, Maia M, Ventura BV, Linden VVD, Araújo EB, Ramos RC, Rocha MAW, Carvalho MDCG, Belfort $\mathrm{R} J \mathrm{r}$, Ventura LO: Ophthalmological findings in infants with microcephaly and presumable intra-uterus Zika virus infection. Arquivos Brasileiros de Oftalmologia 2016, 79:1-3

5. Fernandez MP, Parra Saad E, Ospina Martinez M, Corchuelo S, Mercado Reyes M, Herrera MJ, Parra Saavedra M, Rico A, Fernandez AM, Lee RK, Ventura CV, Berrocal AM, Dubovy SR: Ocular histopathologic features of congenital Zika syndromeocular histopathologic features of congenital Zika syndrome. JAMA Ophthalmol 2017, 135:1163-1169

6. de Paula Freitas B, de Oliveira Dias JR, Prazeres J, Sacramento GA, Ko AI, Maia M, Belfort R Jr: Ocular findings in infants with microcephaly associated with presumed Zika virus congenital infection in Salvador, Brazil. JAMA Ophthalmol 2016, 134:529-535

7. Miranda HA 2nd, Costa MC, Frazao MAM, Simao N, Franchischini S, Moshfeghi DM: Expanded spectrum of congenital ocular findings in microcephaly with presumed Zika infection. Ophthalmology 2016, 123:1788-1794

8. Singh S, Kumar A: Ocular manifestations of emerging flaviviruses and the blood-retinal barrier. Viruses 2018, 10:530

9. Brasil P, Sequeira PC, Freitas AD, Zogbi HE, Calvet GA, de Souza RV, Siqueira AM, de Mendonca MC, Nogueira RM, de Filippis AM, Solomon T: Guillain-Barre syndrome associated with Zika virus infection. Lancet 2016, 387:1482

10. Carod-Artal FJ: Neurological complications of Zika virus infection. Expert Rev Anti Infect Therapy 2018, 16:399-410

11. Miner JJ, Sene A, Richner JM, Smith AM, Santeford A, Ban N, WegerLucarelli J, Manzella F, Ruckert C, Govero J, Noguchi KK, Ebel GD, Diamond MS, Apte RS: Zika virus infection in mice causes panuveitis with shedding of virus in tears. Cell Rep 2016, 16:3208-3218

12. Singh PK, Guest JM, Kanwar M, Boss J, Gao N, Juzych MS, Abrams GW, Yu FS, Kumar A: Zika virus infects cells lining the blood-retinal barrier and causes chorioretinal atrophy in mouse eyes. JCI Insight 2017, 2:e92340

13. Ventura CV, Maia M, Dias N, Ventura LO, Belfort R Jr: Zika: neurological and ocular findings in infant without microcephaly. Lancet 2016, 387:2502
14. Tang H, Hammack C, Ogden SC, Wen Z, Qian X, Li Y, Yao B, Shin J, Zhang F, Lee EM, Christian KM, Didier RA, Jin P, Song H, Ming GL: Zika virus infects human cortical neural progenitors and attenuates their growth. Cell Stem Cell 2016, 18:587-590

15. Salinas S, Erkilic N, Damodar K, Moles JP, Fournier-Wirth C, Van de Perre P, Kalatzis V, Simonin Y: Zika virus efficiently replicates in human retinal epithelium and disturbs its permeability [Erratum appeared in J Virol 2018, 92:e01546-18]. J Virol 2017, $91: \mathrm{e} 02144-16$

16. Roach T, Alcendor DJ: Zika virus infection of cellular components of the blood-retinal barriers: implications for viral associated congenital ocular disease. J Neuroinflammation 2017, 14:43

17. Garcez PP, Stolp HB, Sravanam S, Christoff RR, Ferreira J, Dias AA, Pezzuto P, Higa LM, Barbeito-Andres J, Ferreira RO, Andrade CBV, Siqueira M, Santos TMP, Drumond J, Hoerder-Suabedissen A, de Lima CVF, Tovar-Moll F, Lopes RT, Fragel-Madeira L, Lent R, Ortiga-Carvalho TM, Stipursky J, Bellio M, Tanuri A, Molnar Z: Zika virus impairs the development of blood vessels in a mouse model of congenital infection. Sci Rep 2018, 8:12774

18. Ballabh P, Braun A, Nedergaard M: The blood-brain barrier: an overview: structure, regulation, and clinical implications. Neurobiol Dis 2004, 16 $1-13$

19. Nowakowski Tomasz J, Pollen AlexA, Di Lullo E, SandovalEspinosa C, Bershteyn M, Kriegstein Arnold R: Expression analysis highlights AXL as a candidate Zika virus entry receptor in neural stem cells. Cell Stem Cell 2016, 18:591-596

20. Mladinich MC, Schwedes J, Mackow ER: Zika virus persistently infects and is basolaterally released from primary human brain microvascular endothelial cells. mBio 2017, 8:e00952-17

21. Abbott NJ, Rönnbäck L, Hansson E: Astrocyte-endothelial interactions at the blood-brain barrier. Nat Rev Neurosci 2006, 7:41-53

22. Prebil M, Jensen J, Zorec R, Kreft M: Astrocytes and energy metabolism. Arch Physiol Biochem 2011, 117:64-69

23. Miner JJ, Cao B, Govero J, Smith AM, Fernandez E, Cabrera OH, Garber C, Noll M, Klein RS, Noguchi KK, Mysorekar IU, Diamond MS: Zika virus infection during pregnancy in mice causes placental damage and fetal demise. Cell 2016, 165: 1081-1091

24. Zhao Z, Yang M, Azar SR, Soong L, Weaver SC, Sun J, Chen Y, Rossi SL, Cai J: Viral retinopathy in experimental models of Zika infection. Invest Ophthalmol Vis Sci 2017, 58:4355-4365

25. Shah A, Kumar A: Zika virus infection and development of a murine model. Neurotox Res 2016, 30:131-134

26. Thaker SK, Chapa T, Garcia G Jr, Gong D, Schmid EW, Arumugaswami V, Sun R, Christofk HR: Differential metabolic reprogramming by Zika virus promotes cell death in human versus mosquito cells. Cell Metab 2019, 29:1206-1216.e4

27. Kumar A, Hou S, Airo AM, Limonta D, Mancinelli V, Branton W, Power C, Hobman TC: Zika virus inhibits type-I interferon production and downstream signaling. EMBO Rep 2016, 17:1766-1775

28. Grant A, Ponia SS, Tripathi S, Balasubramaniam V, Miorin L, Sourisseau M, Schwarz MC, Sanchez-Seco MP, Evans MJ, Best SM, Garcia-Sastre A: Zika virus targets human STAT2 to inhibit type I interferon signaling. Cell Host Microbe 2016, 19:882-890

29. Winkler CW, Myers LM, Woods TA, Messer RJ, Carmody AB, McNally KL, Scott DP, Hasenkrug KJ, Best SM, Peterson KE: Adaptive immune responses to Zika virus are important for controlling virus infection and preventing infection in brain and testes. J Immunol 2017, 198:3526-3535

30. Manangeeswaran M, Ireland DD, Verthelyi D: Zika (PRVABC59) infection is associated with $\mathrm{T}$ cell infiltration and neurodegeneration in CNS of immunocompetent neonatal C57B1/6 Mice. PLoS Pathog 2016, 12:e1006004

31. Elong Ngono A, Vizcarra EA, Tang WW, Sheets N, Joo Y, Kim K, Gorman MJ, Diamond MS, Shresta S: Mapping and role of the CD8+ $\mathrm{T}$ cell response during primary Zika virus infection in mice. Cell Host Microbe 2017, 21:35-46 
32. Tang WW, Young MP, Mamidi A, Regla-Nava JA, Kim K, Shresta S: A mouse model of Zika virus sexual transmission and vaginal viral replication. Cell Rep 2016, 17:3091-3098

33. Lazear HM, Govero J, Smith AM, Platt DJ, Fernandez E, Miner JJ, Diamond MS: A mouse model of Zika virus pathogenesis. Cell Host Microbe 2016, 19:720-730

34. Singh PK, Kasetti RB, Zode GS, Goyal A, Juzych MS, Kumar A: Zika virus infects trabecular meshwork and causes trabeculitis and glaucomatous pathology in mouse eyes. mSphere 2019, 4:e00173-00119

35. Harvey K, Tapon N: The Salvador-Warts-Hippo pathway: an emerging tumour-suppressor network. Nat Rev Cancer 2007, 7:182-191

36. Zhao B, Li L, Lei Q, Guan KL: The Hippo-YAP pathway in organ size control and tumorigenesis: an updated version. Genes Dev 2010, $24: 862-874$

37. Zhao B, Wei X, Li W, Udan RS, Yang Q, Kim J, Xie J, Ikenoue T, Yu J, Li L, Zheng P, Ye K, Chinnaiyan A, Halder G, Lai Z-C, Guan K-L: Inactivation of YAP oncoprotein by the Hippo pathway is involved in cell contact inhibition and tissue growth control. Genes Dev 2007, 21:2747-2761

38. Moroishi T, Hayashi T, Pan WW, Fujita Y, Holt MV, Qin J, Carson DA, Guan KL: The Hippo pathway kinases LATS1/2 suppress cancer immunity. Cell 2016, 167:1525-1539.e17

39. Katagiri K, Katakai T, Ebisuno Y, Ueda Y, Okada T, Kinashi T: Mst1 controls lymphocyte trafficking and interstitial motility within lymph nodes. EMBO J 2009, 28:1319-1331

40. Boro M, Singh V, Balaji KN: Mycobacterium tuberculosis-triggered Hippo pathway orchestrates CXCL1/2 expression to modulate host immune responses. Sci Rep 2016, 6:37695

41. Crequer A, Picard C, Patin E, D'Amico A, Abhyankar A, Munzer M, Debre M, Zhang SY, de Saint-Basile G, Fischer A, Abel L, Orth G, Casanova JL, Jouanguy E: Inherited MST1 deficiency underlies susceptibility to EV-HPV infections. PLoS One 2012, 7:e44010

42. Zhang Q, Meng F, Chen S, Plouffe SW, Wu S, Liu S, Li X, Zhou R, Wang J, Zhao B, Liu J, Qin J, Zou J, Feng XH, Guan KL, Xu P: Hippo signalling governs cytosolic nucleic acid sensing through YAP/TAZ-mediated TBK1 blockade. Nat Cell Biol 2017, 19: 362-374

43. Committee for the Update of the Guide for the Care and Use of Laboratory Animals; National Research Council: Guide for the Care and Use of Laboratory Animals. 8th ed. Washington, DC, National Academies Press, 2011

44. Liao JL, Yu J, Huang K, Hu J, Diemer T, Ma Z, Dvash T, Yang XJ, Travis GH, Williams DS, Bok D, Fan G: Molecular signature of primary retinal pigment epithelium and stem-cell-derived RPE cells. Hum Mol Genet 2010, 19:4229-4238

45. Liu Z, Jiang R, Yuan S, Wang N, Feng Y, Hu G, Zhu X, Huang K, Ma J, Xu G, Liu Q, Xue Z, Fan G: Integrated analysis of DNA methylation and RNA transcriptome during in vitro differentiation of human pluripotent stem cells into retinal pigment epithelial cells. PLoS One 2014, 9:e91416

46. Campeau E, Ruhl VE, Rodier F, Smith CL, Rahmberg BL, Fuss JO, Campisi J, Yaswen P, Cooper PK, Kaufman PD: A versatile viral system for expression and depletion of proteins in mammalian cells. PLoS One 2009, 4:e6529

47. Morizono K, Chen IS: Role of phosphatidylserine receptors in enveloped virus infection. J Virol 2014, 88:4275-4290

48. Contreras D, Arumugaswami V: Zika virus infectious cell culture system and the in vitro prophylactic effect of interferons. J Vis Exp 2016, 114:54767

49. Contreras D, Jones MK, Martinez LE, Gangalapudi V, Tang J, Wu Y, Zhao JJ, Chen Z, Wang S, Arumugaswami V: Modeling Zika virus congenital eye disease: differential susceptibility of fetal retinal progenitor cells and ipsc-derived retinal stem cells to Zika virus infection. bioRxiv 2017:128405

50. Langmead B, Trapnell C, Pop M, Salzberg SL: Ultrafast and memory-efficient alignment of short DNA sequences to the human genome. Genome Biol 2009, 10:R25
51. R Core Team: R: a language and environment for statistical computing. Vienna, Austria: R Foundation for Statistical Computing, 2016

52. Love MI, Huber W, Anders S: Moderated estimation of fold change and dispersion for RNA-seq data with DESeq2. Genome Biol 2014, 15:550

53. Benjamini Y, Hochberg Y: Controlling the false discovery rate: a practical and powerful approach to multiple testing. J R Stat Soc Ser B Methodol 1995, 57:289-300

54. Khomtchouk BB, Hennessy JR, Wahlestedt C: shinyheatmap: Ultra fast low memory heatmap web interface for big data genomics. PLoS One 2017, 12:e0176334

55. Wang Y, Xu X, Maglic D, Dill MT, Mojumdar K, Ng PK-S, Jeong KJ, Tsang YH, Moreno D, Bhavana VH, Peng X, Ge Z, Chen $\mathrm{H}$, Li J, Chen Z, Zhang H, Han L, Du D, Creighton CJ, Mills GB, Caesar-Johnson SJ, Demchok JA, Felau I, Kasapi M, Ferguson ML, Hutter CM, Sofia HJ, Tarnuzzer R, Wang Z, Yang L, Zenklusen JC, Zhang J, Chudamani S, Liu J, Lolla L: Comprehensive molecular characterization of the Hippo signaling pathway in cancer. Cell Rep 2018, 25:1304-1317.e1305

56. Zanconato F, Cordenonsi M, Piccolo S: YAP/TAZ at the roots of cancer. Cancer Cell 2016, 29:783-803

57. Wang W, Xiao ZD, Li X, Aziz KE, Gan B, Johnson RL, Chen J: AMPK modulates Hippo pathway activity to regulate energy homeostasis. Nat Cell Biol 2015, 17:490-499

58. Mo JS, Meng Z, Kim YC, Park HW, Hansen CG, Kim S, Lim DS, Guan KL: Cellular energy stress induces AMPK-mediated regulation of YAP and the Hippo pathway. Nat Cell Biol 2015, 17:500-510

59. Garcia D, Shaw RJ: AMPK: mechanisms of cellular energy sensing and restoration of metabolic balance. Mol Cell 2017, 66:789-800

60. Fediuc S, Gaidhu MP, Ceddia RB: Regulation of AMP-activated protein kinase and acetyl-CoA carboxylase phosphorylation by palmitate in skeletal muscle cells. J Lipid Res 2006, 47:412-420

61. Meng Z, Moroishi T, Guan KL: Mechanisms of Hippo pathway regulation. Genes Dev 2016, 30:1-17

62. Zhou D, Zhang Y, Wu H, Barry E, Yin Y, Lawrence E, Dawson D, Willis JE, Markowitz SD, Camargo FD, Avruch J: Mst1 and Mst2 protein kinases restrain intestinal stem cell proliferation and colonic tumorigenesis by inhibition of Yes-associated protein (Yap) overabundance. Proc Natl Acad Sci U S A 2011, 108:E1312-E1320

63. Kandilya D, Maskomani S, Shyamasundar S, Tambyah PA, Shiao Yng C, Lee RCH, Hande MP, Mallilankaraman K, Chu JJH, Dheen ST: Zika virus alters DNA methylation status of genes involved in Hippo signaling pathway in human neural progenitor cells. Epigenomics 2019, 11:1143-1161

64. Mizutani T, Neugebauer N, Putz EM, Moritz N, Simma O, ZebedinBrandl E, Gotthardt D, Warsch W, Eckelhart E, Kantner H-P, Kalinke U, Lienenklaus S, Weiss S, Strobl B, Müller M, Sexl V, Stoiber D: Conditional IFNAR1 ablation reveals distinct requirements of type I IFN signaling for NK cell maturation and tumor surveillance. Oncoimmunology 2012, 1:1027-1037

65. Zhong W, Tian K, Zheng X, Li L, Zhang W, Wang S, Qin J: Mesenchymal stem cell and chondrocyte fates in a multishea microdevice are regulated by Yes-associated protein. Stem Cell Dev 2013, 22:2083-2093

66. Wang L, Luo JY, Li B, Tian XY, Chen LJ, Huang Y, Liu J, Deng D, Lau CW, Wan S, Ai D, Mak KK, Tong KK, Kwan KM, Wang N, Chiu JJ, Zhu Y, Huang Y: Integrin-YAP/TAZ-JNK cascade mediates atheroprotective effect of unidirectional shear flow. Nature 2016, 540:579-582

67. Hussmann KL, Samuel MA, Kim KS, Diamond MS, Fredericksen BL: Differential replication of pathogenic and nonpathogenic strains of West Nile virus within astrocytes. J Virol 2013, 87:2814-2822

68. Cheeran MC, Hu S, Sheng WS, Rashid A, Peterson PK, Lokensgard JR: Differential responses of human brain cells to West Nile virus infection. J Neurovirol 2005, 11:512-524

69. Retallack H, Di Lullo E, Arias C, Knopp KA, Laurie MT, SandovalEspinosa C, Mancia Leon WR, Krencik R, Ullian EM, Spatazza J, Pollen AA, Mandel-Brehm C, Nowakowski TJ, Kriegstein AR, 
DeRisi JL: Zika virus cell tropism in the developing human brain and inhibition by azithromycin. Proc Natl Acad Sci U S A 2016, 113: $14408-14413$

70. Potokar M, Jorgacevski J, Zorec R: Astrocytes in flavivirus infections. Int J Mol Sci 2019, 20:691

71. Huang Z, Wang Y, Hu G, Zhou J, Mei L, Xiong WC: YAP is a critical inducer of SOCS3, preventing reactive astrogliosis. Cereb Cortex 2016, 26:2299-2310

72. Nehme NT, Schmid JP, Debeurme F, Andre-Schmutz I, Lim A, Nitschke P, Rieux-Laucat F, Lutz P, Picard C, Mahlaoui N, Fischer A, de Saint Basile G: MST1 mutations in autosomal recessive primary immunodeficiency characterized by defective naive T-cell survival. Blood 2012, 119:3458-3468

73. Mankouri J, Harris M: Viruses and the fuel sensor: the emerging link between AMPK and virus replication. Rev Med Virol 2011, 21: 205-212

74. Cheng XY, Li YY, Huang C, Li J, Yao HW: AMP-activated protein kinase reduces inflammatory responses and cellular senescence in pulmonary emphysema. Oncotarget 2017, 8: 22513-22523

75. Kumar A, Giri S, Kumar A: 5-Aminoimidazole-4-carboxamide ribonucleoside-mediated adenosine monophosphate-activated protein kinase activation induces protective innate responses in bacterial endophthalmitis. Cell Microbiol 2016, 18:1815-1830

76. Jimenez de Oya N, Blazquez AB, Casas J, Saiz JC, MartinAcebes MA: Direct activation of adenosine monophosphate-activated protein kinase (AMPK) by PF-06409577 inhibits flavivirus infection through modification of host cell lipid metabolism. Antimicrob Agents Chemother 2018, 62:e00360-18

77. Cheng F, Ramos da Silva S, Huang IC, Jung JU, Gao SJ: Suppression of Zika virus infection and replication in endothelial cells and astrocytes by PKA inhibitor PKI 14-22. J Virol 2018, 92: e02019-17

78. Kumar A, Singh PK, Giri S: AMP-activated kinase (AMPK) promotes innate immunity and antiviral defense against Zika virus induced ocular infection. J Immunol 2018, 200(Suppl):50.14

79. Sorrentino G, Ruggeri N, Specchia V, Cordenonsi M, Mano M, Dupont S, Manfrin A, Ingallina E, Sommaggio R, Piazza S, Rosato A, Piccolo S, Del Sal G: Metabolic control of YAP and TAZ by the mevalonate pathway. Nat Cell Biol 2014, 16:357-366

80. Tanaka K, Osada H, Murakami-Tonami Y, Horio Y, Hida T, Sekido Y: Statin suppresses Hippo pathway-inactivated malignant mesothelioma cells and blocks the YAP/CD44 growth stimulatory axis. Cancer Lett 2017, 385:215-224 